\title{
Accretion into Black Hole, and Formation of Magnetically Arrested Accretion Disks
}

\author{
Gennady S. Bisnovatyi-Kogan 1,2,3 \\ 1 Space Research Institute of Russian Academy of Sciences, Profsoyuznaya 84/32, 117997 Moscow, Russia; \\ gkogan@iki.rssi.r \\ 2 National Research Nuclear University MEPhI (Moscow Engineering Physics Institute), Kashirskoe Shosse 31, \\ 115409 Moscow, Russia \\ 3 Moscow Institute of Physics and Technology MIPT, Institutskiy Pereulok, 9, Dolgoprudny, \\ 141701 Moscow, Russia
}

Received: 28 March 2019; Accepted: 31 May 2019; Published: 11 June 2019

\begin{abstract}
The exact time-dependent solution is obtained for a magnetic field growth during a spherically symmetric accretion into a black hole $(\mathrm{BH})$ with a Schwarzschild metric. Magnetic field is increasing with time, changing from the initially uniform into a quasi-radial field. Equipartition between magnetic and kinetic energies in the falling gas is supposed to be established in the developed stages of the flow. Estimates of the synchrotron radiation intensity are presented for the stationary flow. The main part of the radiation is formed in the relativistic region $r \leq 7 r_{g}$, where $r_{g}$ is a $\mathrm{BH}$ gravitational radius. The two-dimensional stationary self-similar magnetohydrodynamic solution is obtained for the matter accretion into $\mathrm{BH}$, in a presence of a large-scale magnetic field, under assumption, that the magnetic field far from the $\mathrm{BH}$ is homogeneous and its influence on the flow is negligible. At the symmetry plane perpendicular to the direction of the distant magnetic field, the dense quasi-stationary disk is formed around $\mathrm{BH}$, which structure is determined by dissipation processes. Solutions of the disk structure have been obtained for a laminar disk with Coulomb resistivity and for a turbulent disk. Parameters of the shock forming due to matter infall onto the disk are obtained. The radiation spectrum of the disk and the shock are obtained for the $10 \mathrm{M}_{\odot} \mathrm{BH}$. The luminosity of such object is about the solar one, for a characteristic galactic gas density, with possibility of observation at distances less than $1 \mathrm{kpc}$. The spectra of a laminar and a turbulent disk structure around $\mathrm{BH}$ are very different. The laminar disk radiates mainly in the ultraviolet, the turbulent disk emits a large part of its flux in the infrared. It may occur that some of the galactic infrared star-like sources are a single $\mathrm{BH}$ in the turbulent accretion state. The radiative efficiency of the magnetized disk is very high, reaching $\sim 0.5 \dot{M} c^{2}$. This model of accretion was called recently as a magnetically arrested disk (MAD). Numerical simulations of MAD and its appearance during accretion into neutron stars, are considered and discussed.
\end{abstract}

Keywords: accretion disk; black holes; magnetic field

\section{Introduction}

The aim of this paper is to present a review of works on the modeling of the accretion into a black hole $(\mathrm{BH})$ of a gas with a large scale magnetic field. Earlier works on this topic made by the author [1,2], have been done using a simplified analytical solutions, along with approximate one-dimensional solutions. The results of these works have been in large confirmed by 2 and 3D numerical simulations, made recently by the members of the group [3-5], which revived the interest to this model under the name magnetically 
arrested disk (MAD). It follows from this review, that extensive numerical simulations made by this group are still not powerful enough, to reproduce all features of the MAD model, which can be studied in the simplified analytical models. An example of it is a strong shock wave formation because of collision with a disk of the gas, falling along magnetic field lines.

\subsection{Black Holes and Accretion}

A BH is formed, if a mass of a collapsing core exceeds the mass limit of the neutron star (NS). A BH is an object with a very strong gravitational field $\varphi_{G} \sim c^{2}$, described by the general relativity. $\mathrm{BH}$ properties are studied in many of monographs (see, for example, References [6-8]). The most important observational property of a $\mathrm{BH}$ is that it does not permit a light escape, so that in vacuum it could be detected only by a black spot (shadow), on a background of twinkling sources (stars). The space between stars and galaxies is filled with a gas that may fall into $\mathrm{BH}$, being heated. The radiation emitted by this gas, could make a $\mathrm{BH}$ to become visible. A powerful accretion and best observational conditions occur when the black hole is in a binary with a normal star and the material flows from this star on to BH. The observed X-ray sources in binaries-candidates for black holes are: CygX-1, LMCX-1, A0620-00, 4U1658-48(GX339-4) and others [9-12].

We consider here models for accretion onto $\mathrm{BH}$ of a magnetized matter. These models are hydrodynamical, that is, the mean free path of particles is assumed to be less than the binary size. For ionized interstellar medium, this is connected with an entanglement of electron and ion trajectories by the magnetic field and in binaries, where the density is high, the mean free path is determined by Coulomb collisions and is also short enough.

\subsection{Accretion of Magnetized Matter}

The magnetic fields play a very important and in some cases a critical role in a possibility of observations of compact objects, NS and BH. A BH may be observed only when matter falls into them, producing radiation of a hot gas.

It was proposed to search for a $\mathrm{BH}$ in binaries, where radiation due to accretion may be large [8]. It was shown in Reference [13] that during a spherically symmetric accretion into a single black hole the transformation of the kinetic energy into radiation is very small in the absence of magnetic field, with the efficiency $\eta=L_{b h} / \dot{M} c^{2}$ of order $\sim 10^{-8}$ [13], $L_{b h}$ is a luminosity and $\dot{M}$ is a mass flux into BH per unit time. Such an object cannot be observed from the Earth. The situation is drastically changes for a magnetized matter falling into $\mathrm{BH}$, when the efficiency strongly increases and luminosity becomes equal to $0.1 \dot{M} c^{2}$ [13]. The account of the magnetic field had been made in Reference [13], for a completely irregular field. The equipartition between the kinetic energy of the falling matter and the magnetic energy during the accretion, was assumed. The condition of equipartition was supported by annihilation of the magnetic field lines or some other dissipation. Such a flow was considered in the Newtonian approximation, with phenomenological account of general relativity by the cut-off of the solution at $r=1.5 r_{g}$, where $r_{g}=2 G M / c^{2}$ is the gravitational radius in Schwarzschild metrics. The approximation of complete irregularity of the magnetic field is not quite realistic, because a characteristic scale of galactic inhomogeneities is $\sim 3 \times 10^{20} \mathrm{~cm}$ [14] and is much greater than the critical accretion radius $r_{c} \sim G M /\left(u^{2}+a_{c}^{2}\right) \sim 10^{14} \mathrm{~cm}$, when $M \approx M_{\odot}, \sqrt{u^{2}+a^{2}} \sim 10^{6} \mathrm{~cm} / \mathrm{s}$ [8]. Here $u$ is velocity of a star relative to the gas cloud and $a_{c}$ is the sound velocity at the critical point of the flow, where it is equal to the infall velocity of the gas.

The exact time-dependent solution is obtained for a magnetic field growth for a spherically symmetric accretion into a BH with a Schwarzschild metric [1], see also Reference [15]. The magnetic field, homogeneous at the initial moment and 'frozen' in the matter, was considered. It was shown in 
Reference $[16,17]$ that the magnetic field of a collapsing star produced by currents inside it, dies with time as $\left(B \simeq t^{-1}\right)$ for a distant observer. At a spherical accretion onto the $\mathrm{BH}$, a frozen magnetic field, according to the exact solution, is growing with time and tends to a quasi-radial structure. The non-stationary behavior of the magnetic field in a stationary flow is connected with infinite conductivity. The magnetic field dissipation for chaotic magnetic structures leads to formation of a stationary flow. In Section 3 estimations are done for the flux properties in the stationary regime. Equipartition is assumed between magnetic and kinetic energies of the infalling matter. The intensity of the magnetic bremsstrahlung radiation of accreting matter and the spectral energy distribution of the radiation, were roughly determined [1] within this assumption. The heating associated with annihilation of the magnetic field was taken into account and the equation of state for a mixture of non-relativistic nuclei and relativistic electrons for $T \geq 6 \times 10^{9} \mathrm{~K}$ was used in addition to suggestions of Reference [13]. It was shown that the velocity of the infalling matter is less than that of free fall and the energy output due to the accretion may approach $\sim 0.3 \dot{M} c^{2}$. The main contribution to the radiation is given by the essentially relativistic region at $r \approx(2-7) r_{g}$. The spectrum of radiation of the accretion of magnetized gas into a single $\mathrm{BH}$, for the chaotic magnetic field in the gas, is considered in Section 3.

When $\mathrm{BH}$ is in the binary, the falling matter has large angular momentum and forms a disk around it. The accretion disk around a Schwarzschild BH radiates $\sim 0.06 \dot{M} c^{2}$ and its luminosity reaches $0.42 \dot{M} c^{2}$ for the limiting Kerr metrics. The magnetic field may influence the spectrum of radiation and the physical processes in the accreting disk. It was suggested also that magneto-rotational instability may be responsible for a generation of the turbulence in the Keplerian accretion disk [18]. An interesting effect was found for a case of a disk accretion onto a $\mathrm{BH}$ in a binary system. In fact, a source with almost black body radiation appears due to gas, falling into $\mathrm{BH}$ from the last stable orbit. Its temperature is about $(2-3) \times 10^{7} \mathrm{~K}$, and luminosity is comparable to that of the whole disk [1]. The presence of rotation diminishes this effect and it is completely absent in the extremal Kerr metric. This may be considered a feature, distinguishing a rotating $\mathrm{BH}$ from a non-rotating one.

The magnetic field is generated in the disk because of thermoelectrical effects. If the matter would not fall into the hole, values of the generated magnetic field could be very large $\sim\left(10^{8}-10^{10}\right) \mathrm{G}$. Falling of matter and large values of conductivity permit the field to grow only up to several tens of gauss [19], see also References [20,21].

\subsection{Accretion in the Presence of a Large-Scale Magnetic Field}

The magnetic field can play an essential role in the establishment of the flow of accretion and in the transformation of the accretion energy into radiation [13,22]. In Reference [2] a stationary two-dimensional self-similar magnetohydrodynamic solution for the accretion flow was obtained, without assuming a weakness of the magnetic field, the structure and the luminosity of the accretion region were investigated. The Newtonian gravity was used and GR effects were taken into account phenomenologically $[13,23,24]$.

It was assumed that the magnetic field far from a $\mathrm{BH}$ is homogeneous and its influence on the flow is negligible. In the plane perpendicular to the direction of the magnetic field, a dense quasi-stationary disk is formed around a $\mathrm{BH}$, which structure strongly depends on dissipative processes. Later, such a model was considered in Reference [25], where it was called a magnetically arrested disk (MAD). The structure of a disk and the spectrum of the outcoming radiation had been calculated in Reference [2] for a laminar disk with the Coulomb mechanism of dissipation and for a turbulent disk. The density in the radial non-magnetised gas flow, with an adiabatic power $\gamma=4 / 3$, increases eight times in the subsonic region, within the interval from infinity to the critical point, where velocity reaches a sound velocity. The magnetic field, frozen in the matter and homogeneous at infinity, increases with time. The approximate law of 
field increase in the subsonic region, neglecting density changes, was obtained in Reference [2] using the equation of induction (the 'freezing' equation) and the relation $v \sim r^{-2}$, as

$$
B_{r}=\left(1+\frac{3 \dot{M}}{4 \pi \rho_{\infty}} \frac{t}{r^{3}}\right)^{2 / 3} B_{0} \cos \theta, \quad B_{\theta}=-\left(1+\frac{3 \dot{M}}{4 \pi \rho_{\infty}} \frac{t}{r^{3}}\right)^{1 / 3} B_{0} \sin \theta
$$

In the supersonic region $\rho \sim r^{-3 / 2}$, the motion is close to the free-fall and the magnetic field increases with time as [1]:

$$
B_{r}=\left(1+\frac{3}{2} \frac{c t \sqrt{r_{g}}}{r^{3 / 2}}\right)^{4 / 3} B_{0} \cos \theta, \quad B_{\theta}=-\left(1+\frac{3}{2} \frac{c t \sqrt{r_{g}}}{r^{3 / 2}}\right)^{1 / 3} B_{0} \sin \theta
$$

The weak magnetic field at infinity increases with time and begins to influence a flow. As a result, in the region where the field attains the value of $B \sim v \sqrt{4 \pi \rho}$, the two-dimensional essentially non-spherically symmetric stationary flow is formed. Equipartition between the magnetic and kinetic energies is established in this flow (see Section 4.1). Initially the stationary flow is formed near $\mathrm{BH}$, because the characteristic time of the magnetic field increase is minimal there. The zone of the stationary flow increases with time and reaches the boundary of the supersonic flow region. The flow in the subsonic region then ceases to be exactly spherically-symmetric. It is clear however, that in the subsonic region the magnetic energy does not exceed the gravitational energy of the matter and the Alfven velocity $v_{A}$ is less than the sound velocity. As a consequence, in the stationary subsonic flow, a mass flux value $\dot{M}$ is close to the value of $\dot{M}$ for the non-magnetized gas. The strength of the magnetic field at the edge of the stationary zone depends on the slowly varying values $\dot{M}$ and critical sonic radius $r_{c}$ and does not depend on the strength of the field at infinity. In what follows we shall neglect their changes. The stationary flow zone increases with time. The stationarity conditions are determined by the hydrodynamic time $t_{s} \sim r / v \sim r^{3 / 2}$, at $v \sim r^{-1 / 2}$ in the supersonic region and $t_{s} \sim r^{3}$ at $v \sim r^{-2}$ in the subsonic region.

Finally, the region of the flow can be divided into three zones, see Figure 1. In a zone around the collapsing star the stationary supersonic flow is established, with a relatively small thermal velocity $v_{T} \ll v \sim v_{A}$. The self-similar solution for this zone is obtained in Section 4.2. In the second zone the flow is also stationary but here a transition from the subsonic flow to the supersonic flow takes place $\left(v_{T} \sim v_{A} \simeq v\right)$. The exact solution in this zone was not obtained analytically. In the farthest zone the field is growing with time, without influence on the flow pattern, which is almost spherically-symmetric, see, for example, Reference [8]. In the subsonic region, which is stationary with respect to the magnetic field, the stagnation zones are formed, in which a matter does not fall into the star and the pressure is balanced by the magnetic field (see Figure 1). The sizes of two inner zones slowly increase with time. In the Section 4.2 a stationary approximate analytic solution for MAD is presented, with application to the $M_{B H}=10 M_{\odot}$, representing collapsing stars in the Galaxy.

Phenomena in the accretion disk supported against gravity by the magnetic field are considered in Section 4. In the next two parts results of numerical simulations of MAD are presented and magnetohydrodynamic phenomena during accretion of matter with a large-scale magnetic field onto a magnetized NS are considered, in connection with some particular X-ray sources. 


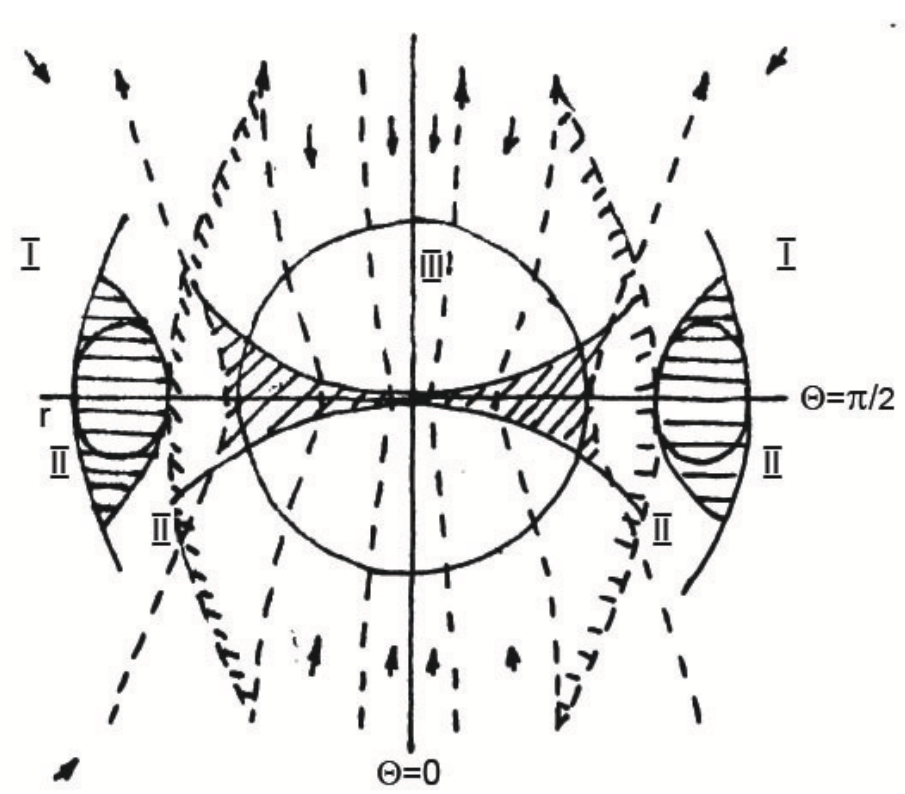

Figure 1. Three zones around a black hole $(\mathrm{BH})$ during accretion of a gas with a frozen magnetic field, homogeneous at infinity. (I) A zone of the stationary hydrodynamic flow with a non-stationary magnetic field. (II) A zone of the stationary flow in which the transition from the subsonic to the supersonic flow takes place. (Ill) A zone of the stationary supersonic flow. Dashed line marks a place where the velocity reaches the critical sound value. At the plane perpendicular to the magnetic field direction at infinity, thin disk forms around a BH. In zone II the disk becomes thicker and merges with the surrounding flow. Arrows in the direction of the flow velocity have opposite signs in the lower and upper parts, the magnetic field has the same direction there. The ring-like stagnation zone formed in the subsonic region of the stationary flow and situated around the symmetry plane is shown by the horizontal strokes. The sizes of two inner zones are slowly increasing with time, see Reference [2].

\section{The Non-Stationary Solution for a Magnetic Field Evolution during a Radial Accretion onto BH}

Let us consider a case, when magnetic field energy density is less than the gravitational one of a gas in the field of a $\mathrm{BH}$ and gas motion is not influenced by magnetic field. We consider stationary radial gas motion in the $\mathrm{BH}$ gravitational field and find the magnetic field dependence on time using the ideal MHD equation $[1,20]$. If the 4 -velocity of the gas $u^{i},(i=0,1,2,3)$, moving in the metric $g_{i k},\left|g_{i k}\right|=g$ is known, that the equation, determining magnetic field are written as [26]

$$
\frac{\partial}{\partial x^{k}} \sqrt{-g}\left(B^{i} u_{k}-B^{k} u^{i}\right)=0,
$$

where $B^{i}$ is the fourfold magnetic field vector, related to the electromagnetic field tensor $F_{l m}$ by the relation

$$
B^{i}=\frac{1}{2 \sqrt{-g}} \varepsilon^{i k l m} u_{k} F_{l m}, \quad B^{i} u_{i}=0,
$$

where $\varepsilon^{i k l m}$ is absolutely antisymmetric four-tensor with only 1 and 0 terms. The condition of orthogonality $B^{i} u_{i}=0$ indicates that there are only three independent components of the magnetic field. The relations (3) and (4) completely determine time-dependent components of the magnetic field for a given $u^{i}, g_{i k}$ and initial field configuration. Let us consider the spherically symmetric stationary flow $u^{i}=\left(u^{0}, u^{r}, 0,0\right)$, 
with initially poloidal magnetic field $B^{i}=\left(B^{0}, B^{r}, B^{\theta}, 0\right)$. In this case Equation (3), taking into account the conditions (4), has a form

$$
\begin{gathered}
\frac{d}{d t}\left(\sqrt{-g} u_{0}^{-1} B^{r}\right)=0, \\
\frac{d}{d t}\left(\sqrt{-g} u_{0}^{-1} B^{\theta}\right)=0, \\
\frac{d}{d t}=\frac{\partial}{\partial t}+\frac{u^{r}}{u^{0}} \frac{\partial}{\partial r} .
\end{gathered}
$$

Equations (5) and (6) are related to the conservation of the magnetic flux along the radial and tangential directions. The solutions of the characteristic system of Equations (5)-(7) is determined by the following integrals

$$
c t-\int \frac{d r}{u^{r} / u^{0}}=c_{1} r_{g}, \quad \sqrt{-g} u_{0}^{-1} B^{r}=c_{2} r_{g}^{2}, \quad \sqrt{-g} u^{r} B^{\theta}=c_{3} r_{g}^{2} .
$$

The time $t$ enters explicitly in the integrals, so the magnetic field at a given space point depends on the time, in the stationary hydrodynamic flow. Let us consider Schwarzschild field metric

$$
d s^{2}=\left(1-r_{g} / r\right) c^{2} d t^{2}-\left(1-r_{g} / r\right)^{-1} d r^{2}-r^{2}\left(d \theta^{2}+\sin ^{2} \theta d \varphi^{2}\right) .
$$

The stationary accretion in the Schwarzschild metric, without a magnetic field, has been considered in Reference [27]. In the supersonic region $r<r_{c}, r_{c}$ is the sound velocity radius, the matter velocity may be approximated by a free fall. The components of the four-dimensional velocity are found from the integrals of motion, related to the conservation of energy $E$ and zero angular momentum $L=0$ [28]

$$
E=m c^{2} g_{00} u^{0}=m c^{2}\left(1-r_{g} / r\right) u^{0}, \quad \sqrt{-g}=r^{2} \sin \theta .
$$

For the matter at rest in the infinity, that is, $E=m c^{2}$, we obtain from Equation (10), using $u^{i} u_{i}=1$, the relations

$$
u^{0}=\left(1-\frac{r_{g}}{r}\right)^{-1}, \quad u^{r}=-\sqrt{\frac{r_{g}}{r}}, \quad u_{0}=1, \quad u_{r}=\sqrt{\frac{r_{g}}{r}}\left(1-\frac{r_{g}}{r}\right)^{-1} .
$$

The physical components of the magnetic field ${ }_{\alpha} B$ are written as

$$
{ }_{r} B=\sqrt{-g_{r r}} B^{r}=\left(1-r_{g} / r\right)^{-1 / 2} B^{r}, \quad{ }_{\theta} B=\sqrt{-g_{\theta \theta}} B^{\theta}=r B^{\theta} .
$$

Consider the initial field in a form of a uniform magnetic field directed along the $z$ axis. In the Schwarzschild coordinate system the initial field components have a form

$$
B^{r}(t=0)=B_{0} \cos \theta, \quad r B^{\theta}(t=0)=-B_{0} \sin \theta(1-x), \quad x=\frac{r_{g}}{r} .
$$

Performing the integrations in (8), with account of (11), and substituting the initial condition (13), we obtain in the parametric form a solution defining the magnetic field

$$
\begin{array}{r}
\frac{c t}{r_{g}}+\frac{2}{3} x^{-3 / 2}+2 x^{-1 / 2}+ \\
+\ln \frac{1-\sqrt{x}}{1+\sqrt{x}}=\frac{2}{3} x_{0}^{-3 / 2}+2 x_{0}^{-1 / 2}+\ln \frac{1-\sqrt{x_{0}}}{1+\sqrt{x_{0}}}, \\
B^{r}=B_{0} \cos \theta \frac{x^{2}}{x_{0}^{2}}, \quad B^{\theta}=-B_{0} \sin \theta \frac{x^{3 / 2}}{r_{g} x_{0}^{1 / 2}}\left(1-x_{0}\right),
\end{array}
$$


where $x_{0}=\frac{r_{g}}{r_{0}}$. To find the physical meaning of the quantity $x_{0}$ we transfer to the comoving coordinates system $(\tau, \varrho, \theta, \phi)$, in which the Schwarzschild metric is written as [28]

$$
d s^{2}=c^{2} d \tau^{2}-\frac{r_{g}}{r} d \varrho^{2}-r^{2}\left(d \theta^{2}+\sin ^{2} \theta d \phi^{2}\right),
$$

with no pathology on the black hole horizon. The connection between Schwarzschild and comoving coordinates $(\tau, \varrho)$ (angle coordinates $\theta, \phi$ are the same) is

$$
\begin{gathered}
c \tau=c t+r_{g}\left[2 x^{-1 / 2}+\ln \frac{1-\sqrt{x}}{1+\sqrt{x}}\right], \\
\varrho=c t+r_{g}\left[\frac{2}{3} x^{-3 / 2}+2 x^{-1 / 2}+\ln \frac{1-\sqrt{x}}{1+\sqrt{x}}\right]
\end{gathered}
$$

After comparison of (14) and (16) we see that the parameter $x_{0}$ is a function of the Lagrangian coordinate $\varrho$ and, therefore, is itself a non-dimensional Lagrangian coordinate, while $r_{0}$ is a dimensional one. For any given time $t$ the matter cannot reach the horizon $(x=1)$, therefore the magnetic field remains equal to zero there [1]. In general ${ }_{\varrho} B$ grows more rapidly than ${ }_{\theta} B$ that is, the asymptotic magnetic field becomes close to the radial one. It is easy to show in the simple Newtonian case, when the equations of the lines of force $d r / B^{r}=r d \theta / B_{\theta}$ may be easily integrated, giving

$$
\left(1+\frac{3}{2} \frac{c t \sqrt{r_{g}}}{r^{3 / 2}}\right)^{2 / 3} r \sin \theta=\text { const. }
$$

At $r \rightarrow \infty$ we obtain the picture of a uniform field $r \sin \theta=$ const. For small $r$ (or as $t \rightarrow \infty$ ) we obtain $\sin \theta \cdot t^{2 / 3}=$ const, that is, a radial magnetic field varying (increasing) with time. In Newtonian limit we obtain the following solution for the evolution with time of the physical components of the magnetic field

$$
x_{0}=x\left(1+\frac{3}{2} \frac{c t \sqrt{r_{g}}}{r^{3 / 2}}\right)^{-2 / 3}, \quad{ }_{Q} B=\left(1+\frac{3}{2} \frac{c t \sqrt{r_{g}}}{r^{3 / 2}}\right)^{4 / 3} B_{0} \cos \theta, \quad{ }_{\theta} B=-\left(1+\frac{3}{2} \frac{c t \sqrt{r_{g}}}{r^{3 / 2}}\right)^{1 / 3} B_{0} \sin \theta .
$$

The solution given by Equations (14) and (18) was obtained for a given radial flow of matter without account of the magnetic field influence on the motion. Actually, the action of the increasing magnetic field terminates its growth and the solution approaches the stationary self-consistent regime. Increase of a magnetic field energy, mainly of the radial component, has a kinetic energy as a source. Therefore in the self-consistent quasi-stationary regime we expect the equipartition between these two energies. For a low-temperature gas the radial flow velocity $v_{r}$ is proportional to the free-fall one and the magnetic energy density is equal to the kinetic energy with gas density $\rho$. Finally, we have the following estimation for the maximum of the radial magnetic field in the flow with a mass flux $\dot{M}$ to BH with a mass $M$ as [1], see numerical estimations in Section 4.3,

$$
v_{r}=\alpha c \sqrt{\frac{r_{g}}{r}}, \rho=\frac{1}{\alpha} \frac{\dot{M}}{4 \pi c r^{2}} \sqrt{\frac{r}{r_{g}}}, \quad\left(B_{r}^{2}\right)_{\max }=\rho v_{r}^{2}=\alpha \frac{\dot{M} c}{r^{2}} \sqrt{\frac{r_{g}}{r}} .
$$

We consider that the lines of force are not gathered in to the centre but are distributed along the disk, forming in the plane $\theta=\pi / 2$ and expanding outward. An approximate diagram of the lines of force is given in Figure 2 from Reference [1]. More detailed considerations of the established flow due to accretion in the presence of a magnetic field is given in the Section 4. Let us estimate the quantities characterising the 
accretion with a chaotic magnetic field and the magnetic bremsstrahlung radiation during such accretion onto a $\mathrm{BH}$.

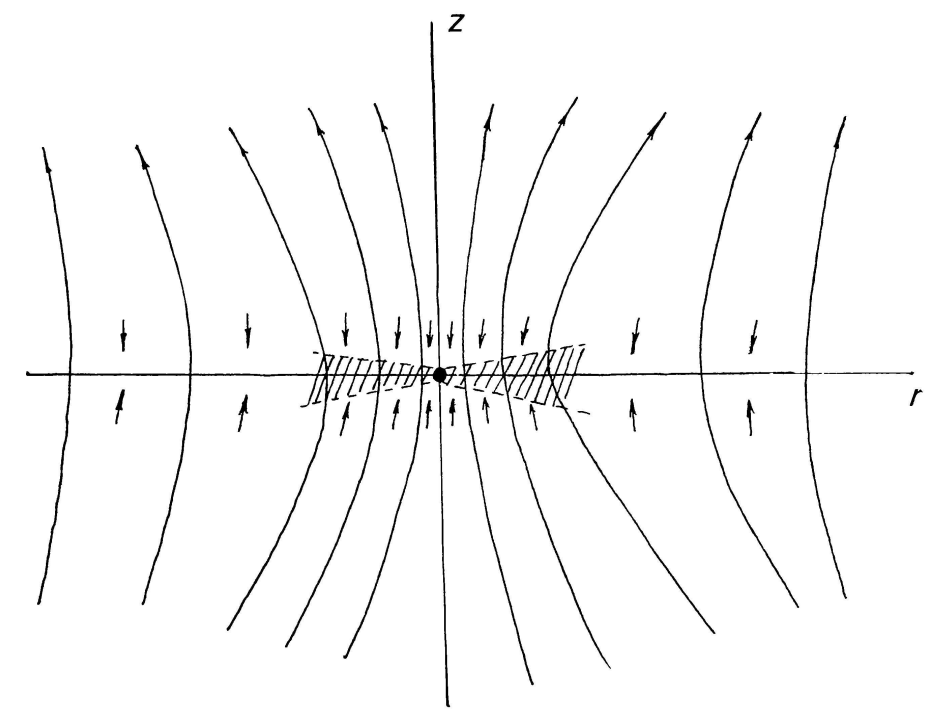

Figure 2. A qualitative picture of a stationary accretion of matter with a large scale magnetic field onto $\mathrm{BH}$, Arrows indicate the direction of motion of the matter. The magnetic field far from the star is in the direction of the $z$-axis. The infalling matter forms a disk in the plane $\theta=\pi / 2$, which slowly settles to the star. In the flow region $E_{B} \sim E_{k i n}$ and rotation is entirely absent from Reference [1].

\section{Radiation Flux and Spectrum at a Radial Accretion of Matter with Chaotic Magnetic Field onto a Black Hole}

In the case of a spherically symmetric accretion, the flow passes through the sound-velocity point at a saddle-like singularity of the system of hydrodynamical equations [29]. The condition for the flow to pass through the sound-velocity point uniquely determines the mass flux $\dot{M}$ and all the flow properties for given $T_{\infty}$ and $\rho_{\infty}$. An adiabatic flow in the point-mass gravitational field can pass through the sound-velocity point only if the adiabatic index $\gamma<5 / 3$. The theory of adiabatic accretion is given in References [29,30] and is similar to the stellar wind theory. We consider here the flows passing through the sound-velocity point and supersonic near the centre of gravity. In presence of a magnetic field more singular points may appear in the accretion flow, connected with magneto-sonic and Alfven velocities, see References [31,32]. In our consideration, the transition through the gas sonic point happens far from $\mathrm{BH}$ and the equipartition zone, where magnetic field influence may be neglected.

Studies of spherically symmetric accretion of interstellar gas onto black holes have shown that, in the presence of a magnetic field frozen in plasma, the efficiency of the kinetic energy conversion into heat approaches $\eta \approx 10 \%$ [13], while in the absence of a field the bremsstrahlung efficiency is $\eta \sim 10^{-8}$. When the gas flows radially, the lines of magnetic force stretch along a radius, $B_{r} \sim r^{-2}$ and the magnetic energy per unit volume $E_{M} \sim B^{2} \sim r^{-4}$ increases more rapidly than the kinetic energy $E_{\text {kin }} \sim \rho v^{2} \sim \dot{M} v / r^{2} \sim r^{-5 / 2}\left(\dot{M}=4 \pi \rho v r^{2}\right.$ is the stationary mass flux, free-fall velocity $\left.v \sim r^{-1 / 2}\right)$. Since the energy $E_{M}$ cannot physically exceed $E_{\text {kin, }}$, it is assumed in Reference [13] that an equipartition of energy $E_{M} \approx E_{\text {kin }}$ is supported by the dissipation of magnetic energy, the excess of which is consumed by plasma heating. This heating was taken into account in Reference [1] and leads to an increase in efficiency $\eta$ to $30 \%$ that may be considered as a realistic estimate under these assumptions. 
If $E_{M} \sim r^{-4}$ is the magnetic field energy with no dissipation, and $E_{M}^{\prime}=E_{\mathrm{kin}} \sim r^{-5 / 2}$ is the energy of the magnetic field in a flow, then an increase of entropy per unit volume along a radius due to the field annihilation in a stationary flow is given by

$$
Q_{M}=\left(\rho T \frac{d S}{d r}\right)_{M}=\left(\frac{d E_{M}}{d r}-\frac{d E_{M}^{\prime}}{d r}\right)_{E_{M}=E_{M}^{\prime}}=-4 \frac{E_{M}}{r}+\frac{5}{2} \frac{E_{M}}{r}=-\frac{3}{2 r} \frac{B^{2}}{8 \pi} .
$$

Consider separately the regions with non-relativistic electrons with

$$
k T \ll m_{\mathrm{e}} c^{2}, \quad \gamma_{1}=5 / 3,
$$

and relativistic electrons with ${ }^{1}$

$$
k T \gg m_{\mathrm{e}} c^{2}, \quad \rho E=\frac{1}{2}\left(3 P+\frac{3}{2} P\right)=\frac{9}{4} P=n P, \quad \gamma_{1}=1+\frac{1}{n}=\frac{13}{9} .
$$

Here, $P_{\mathrm{e}}=P_{\mathrm{p}}=P / 2, \gamma_{1}$ is the adiabatic power, $n$ is the adiabatic index, for simplicity we consider hydrogen plasma. From the energy balance equation

$$
\frac{d E}{d r}-\frac{P}{\rho^{2}} \frac{d \rho}{d r}=\frac{Q_{M}}{\rho}-\frac{\varepsilon_{B}}{v_{r}},
$$

where $\varepsilon_{B}\left(\mathrm{erg} \mathrm{g}^{-1} \mathrm{~s}^{-1}\right)$ is a rate of magneto-bremsstrahlung losses of the Maxwell plasma, with

$$
\frac{B^{2}}{8 \pi}=\frac{1}{2} \rho v_{r}^{2}, \quad v_{r}=\alpha v_{f f}=\alpha \sqrt{\frac{2 G M}{r}}, \overline{B_{\perp}^{2}}=\frac{2}{3} \overline{B^{2}}
$$

and $[1,33]$

$$
\begin{gathered}
\varepsilon_{B}=2 \frac{\mathrm{e}^{2}}{m_{\mathrm{p}} c}\left(\frac{e B_{\perp}}{m_{e} c^{2}}\right)^{2} \frac{k T}{m_{e} c^{2}} \approx 0.46 T B_{\perp}^{2} \mathrm{erg} \mathrm{g}^{-1} \mathrm{~s}^{-1} \text { for } k T \ll m_{e} c^{2} \quad(\mathrm{NR}), \\
\varepsilon_{B}=8 \frac{\mathrm{e}^{2}}{m_{\mathrm{p}} c}\left(\frac{\mathrm{e} B_{\perp}}{m_{e} c^{2}}\right)^{2}\left(\frac{k T}{m_{e} c^{2}}\right)^{2} \approx 3.2 \times 10^{-10} T^{2} B_{\perp}^{2} \mathrm{erg} \mathrm{g}^{-1} \mathrm{~s}^{-1} \text { for } k T \gg m_{e} c^{2} \quad(\mathrm{R}) .
\end{gathered}
$$

We obtain equations for $T(r)$ in the form

$$
\begin{gathered}
\frac{3}{2} \frac{d T}{d r}+\frac{3}{2} \frac{T}{r}+\frac{3}{4} \frac{\alpha^{2} 2 G M}{\mathcal{R}_{g} r^{2}}-1.5 \frac{T \dot{M}}{\mathcal{R}_{g} r^{2}}=0 \quad(\mathrm{NR}) \\
\frac{9}{4} \frac{d T}{d r}+\frac{3}{2} \frac{T}{r}+\frac{3}{4} \frac{\alpha^{2} 2 G M}{\mathcal{R}_{g} r^{2}}-2.2 \times 10^{-10} \frac{T^{2} \dot{M}}{\mathcal{R}_{g} r^{2}}=0 .
\end{gathered}
$$

Here $\mathcal{R}_{g}=2 k / m_{p}$ is the gas constant for ionized hydrogen. For given values of $\rho_{\infty}, T_{\infty}, M$, the mass flux is determined by

$$
\dot{M}=4 \pi \rho v_{r} r^{2}=\frac{10^{32}}{c^{2}}\left(\frac{M}{M_{\odot}}\right)^{2}\left(\frac{\rho_{\infty}}{10^{-24} \mathrm{~g} \mathrm{~cm}^{-3}}\right) \times\left(\frac{T_{\infty}}{10^{4} \mathrm{~K}}\right)^{-3 / 2} \mathrm{~g} \mathrm{~s}^{-1} .
$$

1 Protons are always non-relativistic. 
Neglecting radiation in Equation (26) and adiabatic heating in Equation (27), we obtain the solution in the form

$$
\begin{gathered}
T=2 \times 10^{12} x+2.7 \times 10^{12} x \alpha^{2} \ln \left(\frac{10^{8} x}{T_{\infty} / 10^{4} \mathrm{~K}}\right) \quad(\mathrm{NR}) \\
T=\frac{m_{e} c^{2} 2}{k}+T_{1} \frac{e^{a\left(x-x_{0}\right)}-1}{e^{a\left(x-x_{0}\right)}+1}, \quad T_{1} \gg \frac{m_{e} c^{2} 2}{k} \quad(\mathrm{R}) .
\end{gathered}
$$

Here,

$$
\begin{aligned}
x & =r_{g} / r=\frac{2 G M}{r c^{2}}<1, \\
T_{1} & =2.8 \times 10^{12} \alpha\left(\frac{T_{\infty}}{10^{4}}\right)^{3 / 4}\left(M / M_{\odot}\right)^{-1 / 2}\left(\frac{\rho_{\infty}}{10^{-24}}\right)^{-1 / 2}, \\
a & =1.3 \alpha\left(\frac{M}{M_{\odot}}\right)^{1 / 2}\left(\frac{T_{\infty}}{10^{4} \mathrm{~K}}\right)^{-3 / 4}\left(\frac{\rho_{\infty}}{10^{-24} \mathrm{~g} \mathrm{~cm}^{-3}}\right) .
\end{aligned}
$$

A value of $x_{0}$ slightly depends on $T_{\infty}, \rho_{\infty}$, and for various values of $\alpha$ it is shown in Table 1 . When $x=x_{0}$, we have $T=m_{e} c^{2} 2 / k$ and the solutions (29) and (30) fit to one another. The luminosity and spectrum for this model were calculated in Reference [1]. The luminosity due to magneto-bremsstrahlung is determined mainly by relativistic electrons from (30), giving

Table 1. Dependence of $x_{0}$ on $\alpha$, from Reference [1].

\begin{tabular}{cccc}
\hline$\alpha^{2}$ & 1 & $1 / 3$ & $1 / 10$ \\
\hline$x_{0}$ & $2 \times 10^{-4}$ & $5 \times 10^{-4}$ & $1.2 \times 10^{-3}$ \\
\hline
\end{tabular}

$$
\begin{aligned}
& L_{B}=2.7 \times 10^{31} \alpha^{4}\left(M / M_{\odot}\right)^{3}\left(\rho_{\infty} / 10^{-24} \mathrm{~g} \mathrm{~cm}^{-3}\right)\left(T_{\infty} / 10^{4} \mathrm{~K}\right)^{-3}, \quad a<1 \\
& L_{B}=9 \times 10^{31} \alpha^{2}\left(M / M_{\odot}\right)^{2}\left(\rho_{\infty} / 10^{-24} \mathrm{~g} \mathrm{~cm}^{-3}\right)\left(T_{\infty} / 10^{4} \mathrm{~K}\right)^{-3 / 2}, \quad a \gg 1
\end{aligned}
$$

From comparison of this expression with (28), we find that for a realistic value $\alpha^{2}=1 / 3$ the quantity $\eta=L_{B} / \dot{M} c^{2} \leq 30 \%$. An approximate BH emission spectrum $L_{\omega}\left(L_{B}=\int_{0}^{\infty} L_{\omega} d \omega\right)$ for mass $M_{B H}=$ $10 M_{\odot}$ is given in Figure 3 from Reference [34]. The range with $L_{\omega} \sim \omega^{3 / 5}$ is related to the emission of non-relativistic electrons; at $k T \gg m_{e} c^{2} 2, \hbar \omega \ll k T, L_{\omega} \sim \omega^{1 / 3}$, while at $\hbar \omega \approx \hbar \omega_{B, \max }\left(k T_{1} / m_{e} c^{2} 2\right)^{2} \sim$ $10 \mathrm{eV}$ for $\rho_{\infty}=10^{-24} \mathrm{~g} \mathrm{~cm}^{-3}, T_{\infty}=10^{4} \mathrm{~K}, B_{\max } \sim 10^{5} \mathrm{G}$ an exponential cut-off occurs. 


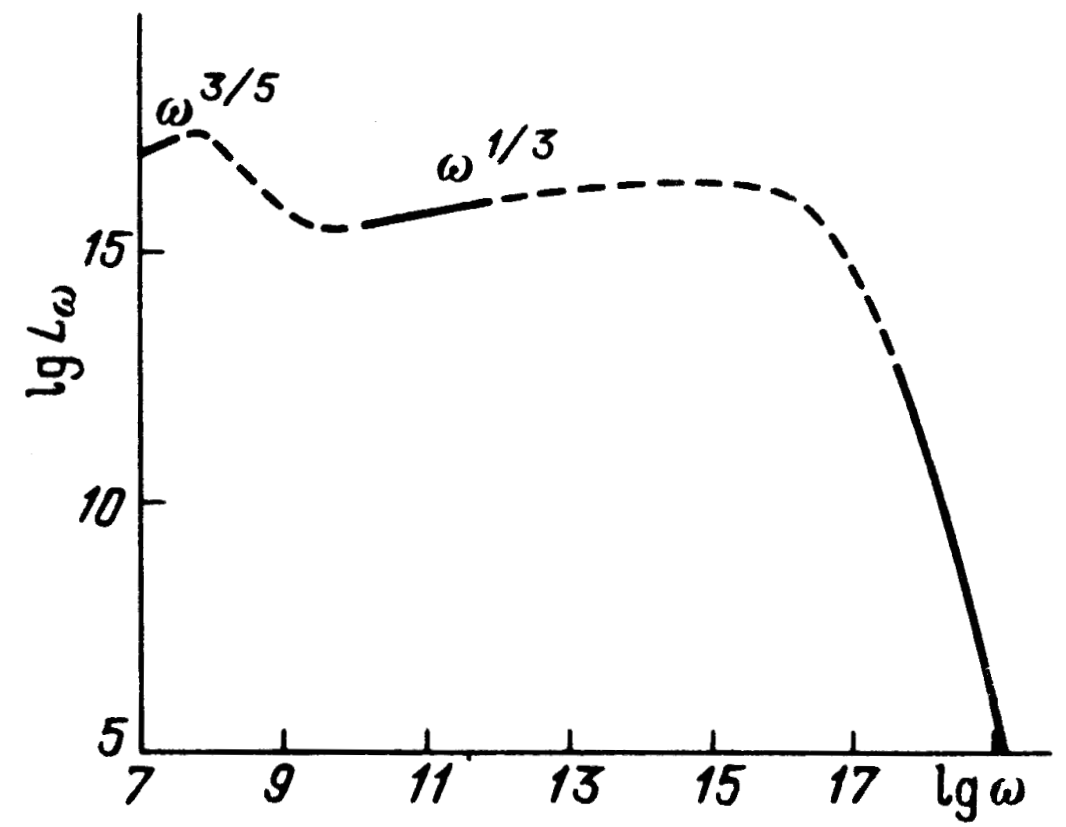

Figure 3. Magneto-bremsstrahlung spectrum of a $\mathrm{BH}$ of $M=10 M_{\odot}$ for a spherically symmetric accretion and random magnetic field at $\rho_{\infty}=10^{-24} \mathrm{~g} \cdot \mathrm{cm}^{-3}, T_{\infty}=10^{4} \mathrm{~K}, \alpha^{2}=1 / 3$. The solid lines represent asymptotic dependencies, dashed lines give extrapolations, from Reference [34].

It was obtained in Reference [1] that the exponential cut-off frequency of bremsstrahlung radiation $\omega_{\text {cut }}$, of thermally heated gas, has the following dependence on accretion parameters

$$
\omega_{\text {cut }} \sim \alpha^{5 / 2} T_{\infty}^{3 / 4} \rho_{\infty}^{-1 / 2} M^{-1}
$$

so for the same surrounding gas the cut-off frequency is inversely proportional to a BH mass. We may conclude therefore, that the observed high energy radiation from a super-massive BH in AGNs is formed due to strong non-thermal heating of electrons around $\mathrm{BH}$ or/and in jets, observed in these objects.

The synchrotron radiation spectrum of a unit volume of relativistic Maxwell plasma is given by [33]

$$
\begin{gathered}
I_{\omega}=\frac{\sqrt{2} \rho e^{2}}{6 \mu_{\mathrm{Z}} m_{\mathrm{p}} c} \omega_{B} z \exp \left[-\left(\frac{9}{2}\right)^{1 / 3} z^{1 / 3}\right], \quad z \gg 1 \\
=\frac{3^{1 / 6}}{\pi} \Gamma(4 / 3) \Gamma(5 / 3) \frac{\rho e^{2}}{\mu_{\mathrm{Z}} m_{\mathrm{p}} c} \omega_{B} z^{1 / 3}, \quad z \ll 1 \\
z=\frac{\omega}{\omega_{B}}\left(\frac{m_{e} c^{2}}{k T}\right)^{2}, \quad I\left(\mathrm{erg} \mathrm{cm}^{-3} \mathrm{~s}^{-1}\right)=\int_{0}^{\infty} I_{\omega} d \omega .
\end{gathered}
$$

The visible magnitude $m_{v}$ for such a $\mathrm{BH}$ is given by [1]

$$
\begin{gathered}
m_{V}=4.8-2.5 \log L / L_{\odot}+5 \ln (R / 10 \mathrm{pc}) \approx 14.1-7.5 \log M / M_{\odot} \\
-2.5 \log \left[\left(\frac{\rho_{\infty}}{10^{-24} \mathrm{~g} \mathrm{~cm}^{-3}}\right)^{3 / 2}\left(\frac{T_{\infty}}{10^{4} \mathrm{~K}}\right)^{-9 / 4}\right]+5 \log \left(\frac{R}{10 \mathrm{pc}}\right) .
\end{gathered}
$$


At increasing of luminosity, connected with an increase of a gas density and (or) $\mathrm{BH}$ mass, the interaction of the outgoing bremsstrahlung radiation flux with an accretion flow is becoming essential. Calculations of accretion into a $\mathrm{BH}$ including the reciprocal effect of radiation had been done in References [35,36]. For a case of accretion onto a NS, a similar effect had been investigated in Reference [37].

\section{Accretion at an Ordered Magnetic Field}

If the characteristic scale of non-uniformity of magnetic field is much larger, that the accretion radius

$$
r_{\mathrm{a}}=\frac{G M}{v_{s}^{2}}, \quad \text { where } v_{s} \text { is the sound velocity in the gas, }
$$

the flow looses its spherically symmetry. For a uniform magnetic field the accretion symmetry is cylindrical. If a $\mathrm{BH}$ is at rest, a stationary pattern of magnetic lines is established, the gas flows along them and forms a disk in the plane of symmetry. A qualitative picture of the flow is shown in Figure 4. At a finite conductivity in the disk a gas infiltrates through force lines of magnetic field towards a BH. The formation process, the structure of a disk supported by a magnetic field and its radiation had been considered in References [2,15].

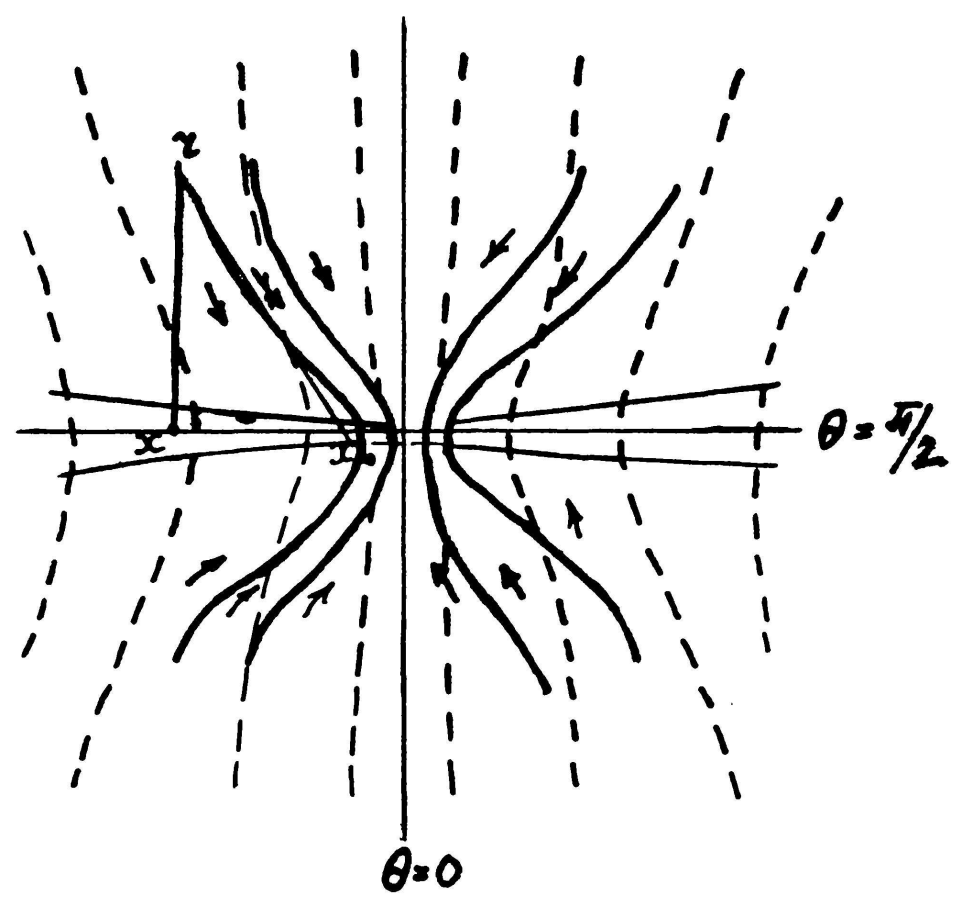

Figure 4. Schematic pattern of magnetic field lines in the matter around a $\mathrm{BH}$ for a field which is uniform at infinity, with the inclusion of distortions due to disk currents. The non-perturbed external magnetic field is shown by dashed lines. The solid lines determine the magnetic field lines, influenced by the azimuthal electrical currents in the disk. The domain of the disk influence is determined by the radius $r\left(x_{0}\right), x\left(x_{0}\right)$, where field perturbations from the disk are small. The arrows indicate a direction of a gas flux velocity, with account of perturbations from the disk, from Reference [2].

\subsection{Self-Similar Solution for the Stationary Flow Outside the Symmetry Plane}

Let us consider a flow in the inner supersonic region, where pressure is negligible and such a flow is essentially directed by the magnetic field. In the axial symmetry case, the stationary picture will be 
two-dimensional. The basic equations for two-dimensional accretion onto a gravitating centre (BH) with mass $M$, of the magnetized gas with perfect conductivity and without pressure are written in the form [2]

$$
\begin{gathered}
v_{r} \frac{\partial v_{r}}{\partial r}+\frac{v_{\theta}}{r} \frac{\partial v_{r}}{\partial \theta}-\frac{v_{\theta}^{2}}{r}=-\frac{G M}{r^{2}}-\frac{B_{\theta}}{4 \pi \rho r}\left[\frac{\partial\left(r B_{\theta}\right)}{\partial r}-\frac{\partial B_{r}}{\partial \theta}\right], \\
v_{r} \frac{\partial v_{\theta}}{\partial r}+\frac{v_{\theta}}{r} \frac{\partial v_{\theta}}{\partial \theta}+\frac{v_{r} v_{\theta}}{r}=\frac{B_{r}}{4 \pi \rho r}\left[\frac{\partial\left(r B_{\theta}\right)}{\partial r}-\frac{\partial B_{r}}{\partial \theta}\right], \\
\frac{1}{r} \frac{\partial}{\partial r}\left(r^{2} \rho v_{r}\right)+\frac{1}{\sin \theta} \frac{\partial}{\partial \theta}\left(\sin \theta \rho v_{\theta}\right)=0, \\
\frac{1}{r} \frac{\partial}{\partial r}\left(r^{2} B_{r}\right)+\frac{1}{\sin \theta} \frac{\partial}{\partial \theta}\left(\sin \theta B_{\theta}\right)=0, \\
v_{r} B_{\theta}-v_{\theta} B_{r}=0 .
\end{gathered}
$$

We assumed that a picture of the flow is stationary $(d / d t=0)$, two-dimensional $\partial / \partial \phi=0$ and the matter falling to a $\mathrm{BH}$ has no angular momentum $v_{\phi}=B_{\phi}=0$. Equations (37) and (38) are the $r$ and $\theta$ components of the Euler equation; (39) is the continuity equation; (40) defines zero divergency of the magnetic field ( $\operatorname{div} \mathbf{B}=0)$ and (41) defines the 'freezing' of the magnetic field, when the flow patterns are parallel to the magnetic field lines. The magnetic force on the right sides of Equations (37) and (38) is perpendicular to the streamlines. The Bernoulli equation is obtained by multiplying (37) by $v_{r},(38)$ by $v_{\theta}$ and combining them. It does not contain the magnetic field: that is,

$$
v_{r} \frac{\partial v^{2}}{\partial r}+\frac{v_{\theta}}{r} \frac{\partial v^{2}}{\partial \theta}=-2 v_{r} \frac{G M}{r^{2}}
$$

We did not find a general solution of the system (37)-(41) but have found a self-similar solution of this system in the form

$$
v_{r}=-\sqrt{2 G M / r} f(\theta), \quad v_{\theta}=\sqrt{2 G M / r} g(\theta), \quad \rho=\rho(\theta), \quad \mathbf{B}=a \rho \mathbf{v}, a=\text { const. }
$$

The last relation (43) is connected with a coincidence of the magnetic field and the streamlines directions, follows from comparison of Equations (39) and (40). Substituting two first relations from (43) into Equation (42) we find that it is satisfied when

$$
f^{2}+g^{2}=1
$$

It is convenient, using (43) and (44), to transfer from $(f, g, B, v)$ to new variables $(z, y)$

$$
f=\cos z, \quad g=\sin z \quad y=\frac{a^{2}}{4 \pi} \rho=\frac{B^{2}}{4 \pi \rho v^{2}} .
$$

Here $y$ is equal to the ratio of the magnetic and kinetic energies. For $y$ and $z$, we have two equations of the first order

$$
\begin{aligned}
& \frac{d y}{d \theta}=y(1-y) \frac{\cot z-\cot \theta}{\sin ^{2} z-y} \cdot \sin ^{2} z \\
& \frac{d z}{d \theta}=\frac{1}{2}-\frac{\sin z \cos z(\cot z-\cot \theta)}{\sin z-y} y
\end{aligned}
$$


A solution for zero magnetic field is $y=0, z=\theta / 2$. Using Equation (39), instead of the third relation (43), we obtain the solution in the form [2]

$$
v_{r}=-\sqrt{2 G M / r} \cos (\theta / 2), \quad v_{\theta}=\sqrt{2 G M / r} \sin (\theta / 2), \quad \rho(r, \theta)=\rho_{0} \Phi\left(\frac{r}{R} \sin ^{2} \frac{\theta}{2}\right) \tan \theta / 2,
$$

where $\Phi$ is an arbitrary function. This solution describes axially symmetric streamline around a gravitational centre of particles flow having an angular momentum at infinity, see, for instance, Reference [38]. For an example, at $\Phi(x)=1 / x$ we have the density distribution $\rho(r, \theta)=\frac{\rho_{0}}{\cos (\theta / 2)} \sqrt{\frac{R}{r}}$, describing the flow of a cold gas, with uniform density at infinity, into rapidly moving gravitational centre. A self-similar solution for such a flow, with a shock wave behind the centre of gravity, was obtained in Reference [39]. We are interested, however, in a solution with the spherically-symmetric distribution of matter at infinity. Deviations from the spherical symmetry around a $\mathrm{BH}$ appear only due to the magnetic field. In a general case the numerical solution of the system (46) and (47) is obtained using an expansion near the singular points of the system. This system have three singular points corresponding to the simultaneous zero's of the numerators and denominators in the right-hand sides of Equations (46) and (47)

$$
\text { 1) } \left.y_{c}=0, z_{c}=k \pi ; \quad \text { 2) } y_{c}=1, z_{c}=\pi / 2+k \pi ; \quad 3\right) y_{c}=\sin ^{2} z, z_{c}=\theta+k \pi, k-0,1,2 \ldots
$$

The first two points are special cases of the third one, when $\theta=0, \pi / 2$ but in a view of simplicity and physical clarity they are listed separately. The case $y_{c}=0, z_{c}=k \pi$ corresponds to a non-magnetized radial flow of cold gas, with the solution $y=0, g=0, f=1, v_{\theta}=0, v_{r}=-\sqrt{2 G M / r}$, describing a free-fall velocity flow from infinity. An equation for the singular point at $\sin ^{2} z_{c}-y_{c}=0$ corresponds to the relation between physical quantities as

$$
v_{\theta}= \pm \frac{B}{\sqrt{4 \pi \rho}}
$$

what determines an equality between $v_{\theta}$ and the Alfven velocity in the singular point. A physically relevant solution is described only by real functions. It was shown in Reference [2], using expansion of the solution in this singular point, that real coefficients of expansion exist only for $y_{c}=1$ and have a complex character at $y_{c}<1$. Therefore, the only physical solution of Equations (46) and (47) goes through the singular point where $v_{r}=0$ and Alfven velocity is equal to the full gas physical velocity. An absence of stationary solutions at $y=\frac{a^{2}}{4 \pi} \rho=\frac{B^{2}}{4 \pi \rho v^{2}}<1$ is related to amplification of weak fields in non-stationary regime. It is worth noting, that in motion of a cold gas a relation between variables in the singular point are valid for the whole flow, therefore we consider the solution with $y \equiv 1$, that is, an equipartition between magnetic and kinetic energies everywhere. In this case only one equation remains corresponding to the radial motion of the matter on the pole. The numerical solution of Equation (51) in the region $0 \leq<\theta \leq \pi / 2$ is given in Table 2 . The density in this solution is constant $\rho=\rho_{0}$ and the velocity $v$ is equal to the Alfven velocity $v_{A}=\frac{B}{\sqrt{4 \pi \rho}}$.

Table 2. Self-similar solution for $y=1$, from Reference [2].

\begin{tabular}{cccccccccc}
\hline$\theta$ & $10^{-3}$ & 0.2 & $0 / 4$ & 0.6 & 0.8 & 1 & 1.2 & 1.4 & $\pi / 2$ \\
\hline$z$ & $7.5 \times 10^{-4}$ & 0.15 & 0.30 & 0.46 & 0.62 & 0.78 & 0.96 & 1.2 & 1.4 \\
\hline
\end{tabular}




$$
\frac{d z}{d \theta}=\frac{3}{2}-\tan z \cdot \cot \theta ; \quad \text { with the boundary condition } z(0)=0,
$$

For small $\theta$ the following expansion goes from Equation (51) $z=3 / 4 \theta+\ldots$. The pattern of the streamlines, coinciding with magnetic force lines is shown schematically by dashed lines in Figure 4 , see Reference [2]. Equation (51) does not change under a mirror transformation $\theta \rightarrow \pi-\theta$ and $z \rightarrow-z$. So, the solution is anti-symmetrically extended to the lower hemisphere $\pi / 2<\theta \leq \pi$, with $v_{r}$ having the same sign as at the upper hemisphere (directed to the centre,) and $v_{\theta}$ changes its sign (see Figure 4). Therefore the plane $\theta=\pi / 2$ occurs to be singular. In this plane, a quasi-stationary disk is formed, in which matter moves to a $\mathrm{BH}$, penetrating through magnetic field lined due to finite electrical conductivity.

\subsection{Stationary Accretion Disk in Presence of a Large-Scale Magnetic Field}

We study a structure of a quasi-stationary magnetized accretion disk using a simplified approach [2]. To obtain an analytic solution for the accretion disk structure supported by a magnetic field is possible only when we neglect centrifugal forces from rotation. Account of both forces in construction of the model is possible only by numerical simulations. Some results of simulations of this model are presented is Section 5, where more references are given. It follows from simulations presented in Reference [4], that in the presence of the vertical magnetic field angular momentum is effectively extracted from the falling flow with a Keplerian rotation on the outer boundary, so that in the inner region around BH magnetic forces exceed the centrifugal barrier. The solution shown below is valid therefore for the inner region around a BH. The extended transition zone between the outer Keplerian accretion disk at $r \geq(100-1000) R_{g}$ and inner, magnetic force dominated region at $r \leq(20-40) R_{g}$ may be studied quantitatively only by numerical simulations.

Let us consider an equilibrium of a non-rotating disk with a balance between magnetic forces and gravity:

$$
\frac{G M \Sigma}{r^{2}}=\frac{1}{c} B_{\theta} I_{\varphi} \approx \frac{2 \pi}{c^{2}} I_{\varphi}^{2} .
$$

Here, $\Sigma=2 h \rho$ is the surface density, $\rho$ is the average density of the gas, $I_{\varphi}$ is a circular electrical current surface density. We have roughly [40]

$$
B_{\theta} \approx B_{r} \approx \frac{2 \pi}{c} I_{\varphi} .
$$

Equilibrium along $z$ axis is supported by a balance between the vertical pressure gradient and gravity

$$
\frac{d P}{d z}=-\frac{\rho G M}{r^{2}} \frac{z}{r}, \quad h \approx\left(\frac{r^{3}}{G M} \frac{P}{\rho}\right)^{1 / 2} .
$$

The disk heating due to extraction of the gravitational energy, is related to a slow motion of the disk into a $\mathrm{BH}$ and due to additional heating by matter falling to the disk along magnetic field lines, at an almost free-fall velocity. Finally we obtain an expression for the energy flux from unit disk surface in the form

$$
F=\frac{G M \dot{M}}{4 \pi r^{3}}\left[1+\frac{1}{2}\left(\frac{r}{R}\right)^{3 / 2}\right] .
$$

From the mass conservation law we obtain an expression for the radial velocity of the disk matter $v_{r d}$ in the form

$$
v_{r d}=-\frac{\dot{M}}{2 \pi r \Sigma}\left[1-\left(\frac{r}{R}\right)^{3 / 2}\right] .
$$


Here $\dot{M}$ is determined by the values at infinity, see Equation (28), $R \approx r_{a}$ using (36). Ohmic dissipation takes place in the disk, leading to gas motion through the magnetic field. The surface electrical current density $I_{\varphi}$ is determined by an equation

$$
\frac{G M \dot{M}}{4 \pi r^{3}}\left[1-\left(\frac{r}{R}\right)^{3 / 2}\right]=\frac{I_{\varphi}^{2}}{4 \pi \sigma}
$$

where $\sigma$ is a conductivity. If the disk is opaque in vertical direction to the radiation, the energy is carried to its surface by a radiative heat conductivity, so that we have approximately

$$
a c T^{4}=\kappa \Sigma F
$$

where $T(r)$ is the mean disk temperature. For an optically transparent disk we have

$$
2 F=\Sigma\left(\varepsilon_{f f}+\varepsilon_{B}\right)
$$

This relation includes plasma bremsstrahlung $\varepsilon_{f f}$ and magneto-bremsstrahlung $\varepsilon_{B}$. Equations (52)-(59) with known functions $P, \kappa, \sigma, \varepsilon_{f f}$ and $\varepsilon_{B}$ on the average disk temperature $T$ and density $\rho$, determine a structure of a non-rotating disk with magnetic field around a $\mathrm{BH}$ [2].

The laminar disk is always optically thick, electrons are non-degenerate and non-relativistic, the pressure is determined mainly by ideal ionized gas $P=\rho \mathcal{R} T$. The conductivity of non-relativistic electrons, due to Coulomb collisions $\sigma_{q}$, neglecting magnetic field dependence, is represented by a simple relation [41]

$$
\sigma_{q}=3 \times 10^{6} T^{3 / 2}\left(\frac{\Lambda}{10}\right)^{-1}
$$

where $\Lambda \approx 10$ is the Coulomb logarithm. Two regions may be separated in such a disk. In an outer region, bremsstrahlung and photo-ionization processes with [2]

$$
\kappa_{f f}+\kappa_{b f} \approx 2 \times 10^{24} \rho T^{-7 / 2} \quad \text { at } k T \ll m_{e} c^{2} ; \quad \kappa_{f f}=5 \times 10^{18} \frac{\rho}{T^{3}} \quad \text { at } k T \gg m_{e} c^{2},
$$

determine the opacity. In the inner one there is a dominance of the opacity due to magneto-bremsstrahlung absorption

$$
\kappa_{B} \approx 40 B^{2} / T^{3}, \quad \text { at } k T \ll m_{e} c^{2} ; \quad \kappa_{B}=4.1 \times 10^{-8} \frac{B^{2}}{T^{2}} \quad \text { at } k T \gg m_{e} c^{2} .
$$

Since the Coulomb conductivity is large, the material penetrates slowly through magnetic force lines and in the stationary case, the disk mass turns out to be large: for a $\mathrm{BH}$ of $M=100 M_{\odot}$ the mass of the stationary disk is $M_{d} \approx 0.2 M_{\odot}$. Most of the disk mass is accumulated in its outer parts. The inner disk is the main source of radiation, the temperature there reaches $10^{8}-10^{9} \mathrm{~K}$, the magnetic field $10^{10}-10^{12} \mathrm{G}$. The disk thickness does not exceed $\sim 0.01$ of the radius.

In the turbulent disk the dissipation is going much more rapidly due to meshing of magnetic field lines and the coefficient of the turbulent electrical conductivity is approximated by an expression [2]

$$
\sigma \approx \sigma_{\text {turb }} \approx \frac{c^{2}}{\tilde{\alpha} 4 \pi h \sqrt{P / \rho}}, \quad \tilde{\alpha}=0.1-0.01
$$


Outer regions of a turbulent disk are transparent for radiation, electrons are non-relativistic there, the gas pressure predominates, and contributions of free-free $\varepsilon_{f f}$ and free-bound $\varepsilon_{f b}$ radiation are comparable with magneto-bremsstrahlung losses $\varepsilon_{B}$ from (24). Here we have for non-relativistic plasma

$$
\varepsilon_{f f}+\varepsilon_{f b} \approx 2 \times 10^{22} \rho T^{1 / 2}
$$

At $\left(M r_{g} / M_{\odot} r\right)=m x \geq 100 \tilde{\alpha}^{2}$ electrons are relativistic and $\varepsilon_{B}$ from Equation (25) greatly exceeds

$$
\varepsilon_{f f}^{\mathrm{rel}} \approx 2 \times 10^{16} \rho T \ln \frac{k T}{m_{\mathrm{e}} c^{2}}
$$

see Reference [2]. We used a connection between opacity and emissivity $\varepsilon=A \sigma T^{4} \kappa$, following from the Kirchhoff's law, in accordance with the corresponding constant for non-relativistic Rosseland $\kappa_{f f}$ and $\varepsilon_{f f}$, calculated in textbooks (see, for example, Reference [42]). The same constant $A=170$ was taken in estimations of $\kappa_{B}$ and for the relativistic case of $\kappa_{f f}$,

The zone of relativistic electrons is narrow in radius because the optical thickness increases rapidly and the disk becomes opaque with decreasing radius. In the disk interiors the gas pressure and electron scattering opacity,

$$
\kappa_{e s}=0.19\left(1+X_{H}\right), \quad X_{H} \text { is the hydrogen mass fraction in accreting matter, }
$$

predominate for $10<m x<1000$, while for $m x>1000$ the pressure is radiation dominated. The mass of a turbulent disk is always small because of a large dissipation and rapid infiltration of gas through the magnetic field lines. Note the non-monotonic character of the function $T(r)$ connected with transition of a transparent disk into an opaque one with decreasing $r$.

The choice between laminar or turbulent disk models could be done after investigation of the stability of the disk. The calculations carried out in Reference [40] show that the azimuthal currents in the disk tend to stabilize it against fragmentation. Therefore, we cannot exclude here a possibility of a laminar disk. In this sense we find a significant difference between the magnetically supported disk and the keplerian disk at accretion in binary systems, where differential rotation in combination with magnetic field always produces a turbulence, needed to explain observations. Relations describing a distribution of disk parameters are given in Reference [2] for various disk regions in the turbulent and laminar disk with a Coulomb conductivity.

For a laminar disk assumptions about the thinness $(h / r \ll 1)$ and quasi-stationarity of the disk with $v_{r} \ll \sqrt{2 G M / r}$ is fulfilled only if its mass $M_{D}$ is not too large. The restriction on the disk mass $M_{D}$ appears because the stationary disk mass is rapidly increasing with a $\mathrm{BH}$ mass, $\mathrm{M}$ and for a reasonable $\dot{M}$, it takes a longer time to establish the quasi-stationary density distribution. Estimations have shown, see Reference [2], that for $M>100 M_{\odot}$ the stationarity is reached after a time, exceeding the age of the universe $\tau_{u} \approx 5 \times 10^{17}$ c. For a large $M$ it is mainly collecting in the external regions of the accretion disk.

The turbulent disk structure is very different. In the laminar Coulomb disk the temperature continuously increases to the centre, while in the turbulent disk its distribution is more complicated. In the external parts the disk is transparent, the energy losses are determined mainly by free-free transitions and the temperature rises towards to the centre. Then the non-relativistic magneto-bremsstrahlung processes becomes important and the temperature increases until the electrons become relativistic. Then in the ultra-relativistic condition the temperature in not growing and if the masses are sufficiently large $\left(M>10 M_{\odot}\right)$ an internal nontransparent region exists where the electrons are non-relativistic again and the temperature increases to the centre [2]. For given values of $\rho_{\infty}, T_{\infty}, M$, the mass flux is determined by Equation (28). 


\subsection{High Energy Radiation from the Shock on the Disk Surface and Radiation Spectrum}

In the quasi-stationary picture of MAD a matter feeds the accretion disk from two sources. The main gas is coming from extended space in the form of accretion disk, diffusing through the magnetic "wall" due to finite conductivity. In the presence of rotation the gas comes in the form of a Keplerian accretion disk. In a stationary state the poloidal magnetic field almost reaches an equipartition with the kinetic energy of the falling matter and gas moves along magnetic field lines in both hemispheres, until it collides with the existed accretion disk, see Figure 4. The standing shock front is formed in this collision and falling gas joins the accretion disk, increasing the mass flux with approach to a $\mathrm{BH}$. The total mass flux $\dot{M}$, arrived from infinity, is divided between these two sources. In absence of rotation the disk structure starts to form [2] near the accretion radius $r_{a} \equiv R$, giving the distributions presented in Equations (36) and (55)-(57).

The strong deviations from the spherical-symmetric flow, due to large scale magnetic field structure, are essential for formation of a hard component of the radiation in this disk. Indeed, the collision of matter falling along magnetic field lines on the disk surface leads to formation of a shock wave, in which the kinetic energy of infalling matter is transformed into heat. The shock wave is apparently collision-less, with a thin front [33]; therefore, the temperature of the matter, in presence of ultra-relativistic electrons and non-relativistic protons is determined by the relation

$$
\frac{9}{2} k T=\frac{m_{p} v^{2}}{2} .
$$

The energy released in the shock is determined by the gravitational potential at the place of a shock formation and is equal to (see Reference [2])

$$
L_{s h} \simeq \int_{1.5 r_{g}}^{\infty} \frac{d \dot{M}}{d r} \frac{G M}{r} d r
$$

The dependence $\dot{M}(r)$ was obtained in Reference [2], from what it follows, that the hard radiation from the shock $L_{s h}$ contains only a small part of the luminosity, so that for $M_{B H}=10 M_{\odot}$ there is $L_{s h} / L \simeq 10^{-4}$. The hard radiation spectrum from the shock is estimated in Reference [2] for $M_{B H}=10 M_{\odot}$. It has an almost power law, with an exponential cut-off at frequency $\omega \approx 3 \times 10^{23} \mathrm{~s}^{-1}$, corresponding to the energy about $500 \mathrm{MeV}$.

As the gravitational energy always has time to convert into heat during stationary disk accretion, the black-hole luminosity for a minimum radius of $\sim 1.5 r_{g}$ is

$$
L \approx \frac{G M \dot{M}}{r_{g}}=\frac{1}{2} \dot{M} c^{2}=5 \times 10^{31}\left(\frac{M}{M_{\odot}}\right)^{2}\left(\frac{\rho_{\infty}}{10^{-24} \mathrm{~g} \mathrm{~cm}^{-3}}\right) \times\left(\frac{T_{\infty}}{10^{4} \mathrm{~K}}\right)^{-3 / 2} \mathrm{erg} \mathrm{s}^{-1}
$$

with $\dot{M}$ from Equation (28), determining very high efficiency of this accretion regime, which was recently named as magnetically arrested disk (MAD) [25]. The emission spectrum of an opaque disk is related to its effective temperature determined locally by

$$
\frac{a c}{4} T_{\mathrm{ef}}^{4}=F
$$

when the shock energy is supposed to be completely thermalized. Hence, using Equation (55), we have for the inner parts of the disk around a BH with $r \ll R$, where the most part of the energy is released,

$$
T_{\mathrm{ef}} \sim r^{-3 / 4}
$$


After integration over the disk surface with the temperature distribution (71), we obtain the spectral distribution

$$
L(\omega) \sim \omega^{1 / 3},
$$

with an exponential cut-off in the soft $X$-ray region, at $\hbar \omega \sim k T_{\max }, T_{\max } \approx 7 \times 10^{5} \mathrm{~K}$ for $M=10 M_{\odot}$. So, despite a substantial differences in flow patterns in cases of a random and ordered magnetic fields, the spectrum of the optically thick disk is similar to the random field case, and the relation (35) for $m_{v}$ is valid in such a case as well. A turbulent disk has a large transparent region with magneto-bremsstrahlung in the infrared range that may be comparable in power with the ultraviolet and soft X-ray emission of opaque disk interiors. In the case of accretion with a large scale magnetic field a small, almost power-law hard tail is present, continuing until $\sim 500 \mathrm{MeV}$. Such a tail is absent in the case of a spherical accretion with a fully chaotic magnetic field.

If the accreting gas has an intrinsic angular momentum, the magnetized disk generates electric fields $[40,43]$ of the strength $E \approx-(v / c) B$. In this field the electrons are accelerated up to energies $\varepsilon \approx R(v / c) B e \approx 3 \times 10^{4}\left[B /\left(10^{7}\right.\right.$ Gauss $\left.)\right]$ Mev where $v / c \approx 0.1$ and $R \approx 10^{7} \mathrm{~cm}$ is the characteristic scale. In a field $B \approx 10^{7}$ Gauss, such electrons generate synchrotron radiation with energies up to $\approx 10^{5} \mathrm{keV}$, which flux may be much larger than the hard radiation flux from the shock. Like in pulsars, it would be possible here for $e^{+} e^{-}$pairs formation, which participate in the synchrotron radiation. Such a mechanism is likely to act in Cyg X-1, galactic nuclei $[44,45]$ and is analogous to the unipolar mechanism proposed for explaining the pulsar radiation [46].

Using the MAD solution the gas rotation is small in the inner part of the accretion disk. In this case a formation of the electrical field may be connected with $\mathrm{BH}$ rotation and processes inside the ergosphere, where matter rotation becomes inevitable. This condition was considered in Reference [44], for the extraction of the rotational energy from the $\mathrm{BH}$.

\section{Numerical Simulations of Magnetically Arrested Disk}

Two- and three-dimensional MHD simulations had been performed in Reference [4] for investigation of the dynamics and structure of magnetically arrested disk (MAD), in presence of a rotation. It was obtained. that MAD was formed in accretion flows, carrying inward large-scale poloidal magnetic fields. Because of rotation, the streams take spiral shapes. The jets formation takes place in MAD, because of the interaction of the spiraling accretion flow with the vertical magnetic bundles, twisted around the axis of rotation. Ideal non-stationary MHD 3-D equations have been used with an ideal gas equation of state

$$
P=(\gamma-1) \rho \varepsilon,
$$

at adiabatic power $\gamma=5 / 3$. Energy equation was included in the system because a growth of the entropy due to numerical resistivity and viscosity and in possible MHD shocks. Self-gravity of gas was neglected and a pseudo-Newtonian approximation [47] was employed for the $\mathrm{BH}$ potential.

$$
\Phi=-\frac{G M}{R-R_{g}}, \quad R_{g}=\frac{2 G M}{c^{2}} \text { is the } \mathrm{BH} \text { gravitational radius. }
$$

Radiatively inefficient accretion disk with poloidal magnetic fields was considered, without a cooling term in the energy equation, similar to Reference [48], see however, Reference [49]. The MHD equations have been solved by using the time explicit Eulerian finite-difference scheme, which is an extension to MHD of the hydrodynamic piecewise-parabolic method by Reference [50]. Spherical coordinates $(R, \theta, \phi)$ were used. The 3D numerical grid had $182 \times 84 \times 240$ zones in the radial, polar and azimuthal directions, respectively. The radial zones were spaced logarithmically from $R_{i n}=2 R_{g}$ to $R_{\text {out }}=220 R_{g}$. Two polar 
cones with an opening angle of $\pi / 8$ were excluded. So, the polar domain extends from $\theta=\pi / 16$ to $15 \pi / 16$. The grid resolution in the polar direction was gradually changed from a fine resolution around the equatorial plane to a coarse resolution near the poles, with a maximum-to-minimum grid size ratio $\approx 3$. The azimuthal zones cover uniformly the full $2 \pi$ range in $\phi$, see more detail of the numerical method in Reference [4]. Initially, the computational region was filled with a very low-density, non-magnetized gas. The simulations were started in 2D, assuming axial symmetry, with an injection of mass with a finite entropy and Keplerian angular momentum in a slender torus, located in the equatorial plane at $R_{i n j}=210 R_{g}$. Simulations without the magnetic field were finished after formation of a steady thick torus, with the inner edge at $R_{i n} \approx 150 R_{g}$ and the outer part is truncated at $R_{\text {out }}$. The torus contained a constant amount of mass and is in dynamic equilibrium: all injected mass flows outward through $R_{\text {out }}$ after circulation inside the torus. No accretion flow was formed at this point. This hydrodynamic, steady, thick torus was used as an initial configuration for the MHD simulations.

The MHD simulations were started at $t=0$ from the steady, thick torus by starting injection of a poloidal magnetic field into the slender torus at $R_{i n j}$. The numerical procedure for the field injection is described in Reference [48]. The entire volume of the thick torus is filled by the field during about one orbital period, $t_{o r b}$, estimated at $R_{i n j}$. Starting from the moment, $t \simeq t_{o r b}$, formation of the accretion flow begins as a result of redistribution of the angular momentum in the torus due to magnetic forces. Three models were calculated with $\beta_{\text {ing }}=10,100,1000$, which is the ratio of the gas pressure to the magnetic pressure at $R_{i n j}$. After beginning of the accretion an evolution of disks is governed mainly by magnetic forces produced by the poloidal field component. This component is advected inward with the accretion flow and generates strong toroidal magnetic fields, because of the Keplerian rotation, localized above and below the midplane. These toroidal fields form a highly magnetized disk corona with a typical $\beta \sim 0.01$. Due to the permanent mass injection and magnetic field accumulation of a poloidal field happens in the central parts of the accretion disk where MAD is formed. The computational results for $\beta_{\text {ing }}=100$ from Reference [4] are given in Figure 5. MAD formation in the central parts is clearly visible in this Figure. Note, that in the quasi-Newtonian approximation magnetic flux after finite time goes through the horizon. In the GR consideration the distant observer will not see the penetration of the magnetic flux through the horizon, because it take for him an infinite time to reach the horizon for the magnetic flux, as well as for the matter [8]. It does not change the conclusion about a very high efficiency of MAD for producing radiation flux, which is coming from the accretion disk itself. at the finite distance from the horizon.

3-D finite conductivity MHD simulations of the cooling instability in optically thin hot BH accretion flows had been studied in Reference [51] assuming the pseudo-Newtonian gravitational potential [47] and bremsstrahlung cooling. It was found that the cooling instability changed the accretion flow from an optically thin, hot, gas pressure-supported state to a cooler, quasi-steady state MAD structure. The stability of the MAD disk was examined analytically and it was found to be thermally and secularly stable. 3D simulations of accreting BHs had been performed in Reference [5], using time-dependent, non-radiative, general-relativistic, magnetohydrodynamic equations. The main difference from Reference [4] was using full GR equations instead of the pseudo-Newtonian potential [47]. In these simulations a large amount of magnetic flux was transported to the centre, remains outside a $\mathrm{BH}$, impedes accretion and leads to a magnetically arrested disk. Powerful outflows have been found, similar to Reference [4]. An efficiency $\eta$ of transformation of the gravitation energy of accreted mater into the out-flowing energy in jets and winds, depended on a BH spin parameter in the Kerr metrics $a$. It changed from $\eta \approx 30 \%$ for $a=0.5$, up to $\eta \approx 140 \%$ for $a=0.99$, what is an indication to additional energy supply from the rotational energy of $\mathrm{BH}$. The energy extraction from a spinning BH could happen only via the Penrose mechanism [52], generalized for the presence of a magnetic field in Reference [44,53]. 

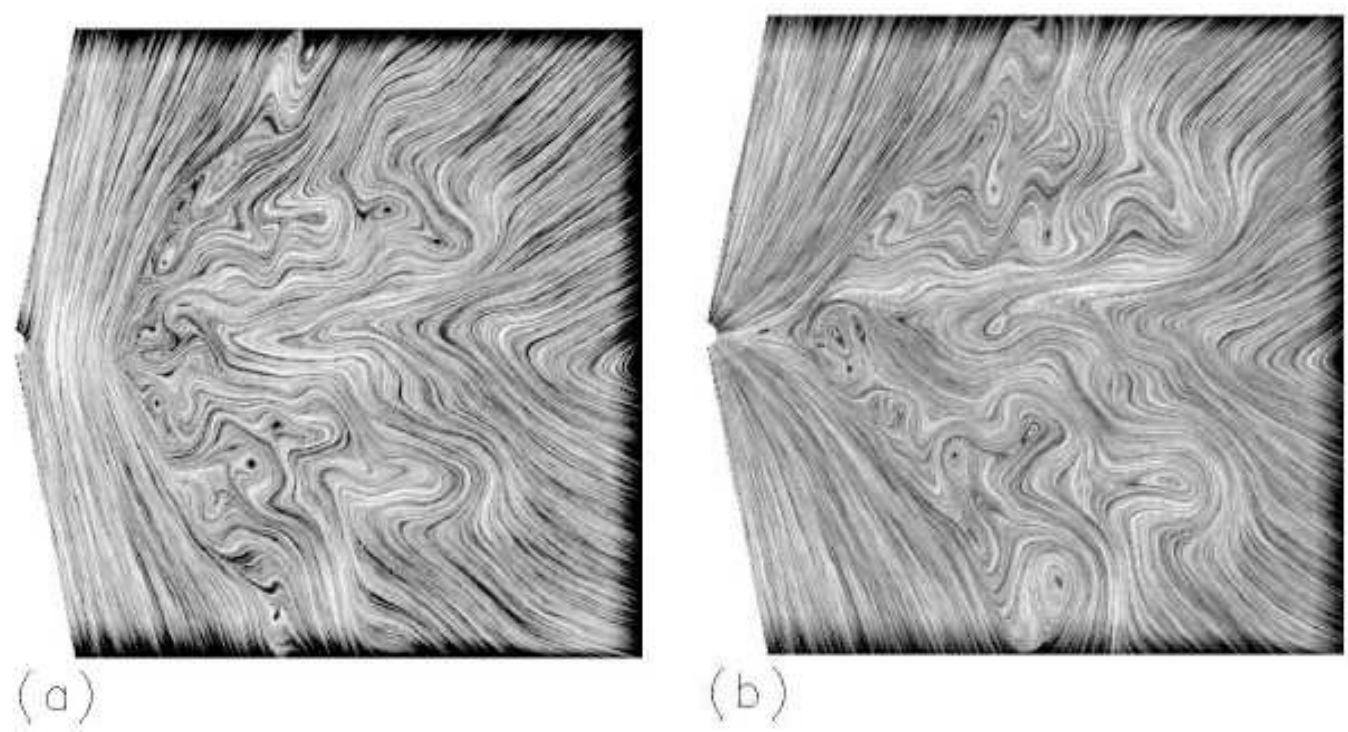

Figure 5. Snapshot of magnetic lines in model with $\beta_{\text {ing }}=100$, at two subsequent moments. The $\mathrm{BH}$ is located on the left and small open circle corresponds to the inner boundary around the black hole at $R_{i n}=2 R_{g}$. The axis of rotation is in the vertical direction. The domain in the figure has a radial size $100 R_{g}$ along the equatorial plane and represents a fraction of the full computational domain with $R_{o u t}=220 R_{g}$. The poloidal field lines lying in the meridional plane are shown. The accretion disk transports the vertical magnetic flux inward, which is accumulated in the vicinity of a BH. Small-scale magnetic loops are the result of turbulent motions in the disk and disk corona. (a) Period of accretion, in which most of the accumulated magnetic flux is outside the black hole horizon. (b) Accretion period, in which all the accumulated flux goes through the horizon, [4].

Equilibrium of two-temperature optically thin MAD disk was studied in Reference [54] using equations averaged over the thickness, with pseudo-Newtonian $\mathrm{BH}$ gravitational potential [47]. Outflows had not been included into consideration. The first Magnetically Arrested Disc simulations with two-temperature, radiative, general relativistic magnetohydrodynamics were performed in Reference [3] in 3-D modeling. Mass accretion rates of the simulations were scaled to match the luminosity of the accretion flow around the super-massive black hole in M87. Several mechanisms of electron heating were considered. The main features of the numerical solution resemble that produce in Reference [4,5].

The stationary picture of the accretion disk around a $\mathrm{BH}$ is formed due to violation of the ideal MHD condition and finite conductivity of a disk matter. The magnetic flux growth stops, when rate of matter penetration trough the magnetic field lines balance the accretion gas flux from large distances. The particular structure of the MAD depends strongly on electrical conductivity of the disk matter. Laminar Coulomb and turbulent electrical conductivities were investigated in Reference [2], see Section 4. It was shown, that only phenomenological turbulent conductivity coefficient, derived in Reference [2], gives a physically relevant stationary solution of MAD. Numerical simulations using ideal MHD [3-5], may be used only for limited period of time and simulations with a finite electrical conductivity [51] permitted to obtain a physically realistic stationary solution.

\section{Magnetized Disk Levitation and Mad}

Many interesting effects appear in consideration of the MAD around neutron stars. In this case the MAD is restricted by the star surface or by the Alfven radius in magnetized stars, where the pressure of its magnetic field is equal to the large scale magnetic field pressure in the disk. This model was considered in 
several papers with an application to explain observational properties of different objects. It was called as magnetic-levitation accretion scenario [55]. Consider several objects where this model was applied for modeling the observational data.

1. The spin-down mechanism of accreting neutron stars was discussed in Reference [56,57], with an application to the X-ray pulsar GX301-2, showing rotation period $P_{s}=685 \mathrm{~s}$. It was obtained that the maximum possible spin-down torque applied to a NS from the accretion flow can be evaluated as $K_{s d}^{(t)}=\mu^{2} /\left(r_{m} r_{c o r}\right)^{3 / 2}$. Here $r_{c o r}=\left(G M_{n s} / \omega_{s}^{2}\right)^{1 / 3}$ is the co-rotation radius of a NS spinning at angular velocity $\omega_{s}=2 \pi / P_{s}, \mu$ is its dipole magnetic moment, $r_{m}$ is the radius of the neutron star magnetosphere (Alfven radius). The spin-down rate of a NS in GX301-2 can be explained if the magnetosphere radius of the neutron star is smaller than its canonical value. The magnetosphere radius was calculated in Reference [56] considering the mass-transfer in the binary system in the model of the magnetic-levitation accretion scenario. It was shown, that the spin-down rate of a NS expected within this approach is in a good agreement with that derived from observations of GX301-2. Other pulsars of this type were considered in References [58,59].

2. Observations of the X-ray pulsar $4 \mathrm{U} 2206+54$ during more than 15 years have shown that its period, which is now $5555 \pm 9 \mathrm{~s}$, is rapidly increasing. Such a behavior is difficult to explain inside traditional scenarios for the spin evolution of compact stars. It was shown [60], that the observed spin-down rate of a NS in $4 \mathrm{U} 2206+54$ is in a good agreement with the value expected in a magnetic-accretion scenario, if the magnetic field of the accretion stream affects the geometry and type of flow. A NS in this case accretes material from a dense gaseous slab with a small angular momentum, which is kept in equilibrium by the magnetic field of the flow. A magnetic accretion scenario can be realized in $4 U 2206+54$ if the magnetic field strength at the surface of the optical counterpart to a NS is higher than $70 \mathrm{G}$. The magnetic field at the surface of the neutron star in this scenario is $\sim 4 \times 10^{12} \mathrm{G}$, in agreement with estimates based on an analysis of X-ray spectra of the pulsar.

3. Growth of the NS period of in the young Be/X-ray long-period pulsar SXP 1062 was considered in Reference [61]. The observed period is about $1062 \mathrm{~s}$ and the star is spinning down at the rate $\sim-2.6 \times 10^{-12}$ $\mathrm{Hz} / \mathrm{s}$. It was shown that all of the conventional accretion scenarios meet big difficulties in explaining the rapid spin-down of this pulsar. It was shown in References [1,2,48], that these difficulties can be avoided within the magnetic accretion scenario, where a NS is assumed to accrete from a magnetized wind. The spin-down rate of the pulsar can be explained within this scenario provided the NS surface magnetic field is $B_{*} \sim 4 \times 10^{13} \mathrm{G}$. It happens, that the age of the pulsar in this case lies in the range $(2-4) \times 10^{4} \mathrm{yr}$, which is consistent with observations.

4. A scenario of the formation of isolated X-ray pulsars was discussed in References $[55,62]$ with an application to the object 1E 161348-5055. This moderately luminous, $10^{33}-10^{35} \mathrm{erg} / \mathrm{s}$ pulsar with a relatively soft spectrum, $k T \sim 0.6-0.8 \mathrm{keV}$, is associated with an isolated neutron star, which is located near the centre of the young $(\sim 2000 \mathrm{yr})$ compact supernova remnant RCW 103 and rotates with a period of $6.7 \mathrm{~h}$ and a slowly decreasing frequency $\left(|\dot{v}| \leq 2.6 \times 10^{-18} \mathrm{~Hz} / \mathrm{s}\right)$. It was shown that at the present epoch the NS is in the accretor state. The parameters of the source emission can be explained in terms of the magnetic-levitation accretion scenario in which a star with the surface magnetic field of $10^{12} \mathrm{G}$ accretes matter onto its surface from a non-Keplerian magnetic fossil disk, at the rate $10^{14} \mathrm{~g} / \mathrm{s}$. A neutron star could evolve to this state in a High-Mass X-ray Binary (HMXB), which had disintegrated during the supernova explosion powered by the core-collapse of its massive component. A life-time of an isolated X-ray pulsar, formed by this way, can be of about few thousand years.

5. A behavior of Anomalous X-ray Pulsars (AXPs) and Soft Gamma ray Repeaters (SGRs) was considered in Reference [63], in a scenario with fall-back magnetic accretion onto a young isolated neutron star. The X-ray emission of the pulsar in this case originates due to the accretion of matter onto the surface 
of a NS from a magnetic slab surrounding its magnetosphere. The spin-down rate of a NS is expected in this picture to be close to the observed value. Such NSs should be relatively young. The pulsar's activity in gamma-rays is related to its relative youth and is enabled by energy stored in a non-equilibrium layer $[64,65]$, located in the crust of a low-mass NS. Outbursts are probably triggered by instability developing in the region where the accreted matter is accumulated.

There are also applications of the MAD model to different astronomical objects, containing a $\mathrm{BH}$.

1. Simulations of the axisymmetric MAD disk - jet system in the pseudo-Newtonian gravitational potential [47] and an approximate treatment of the derivative over $z$ - coordinate, with account of different heating and cooling mechanisms in the disk and jet, had been done in Reference [66]. The magneto-centrifugally driven outflows from the disk threaded by the large scale open magnetic field was obtained. The results have been used for a unified classification of blazars.

2. 2.5-D simulations of the geometrically thick, sub-Keplerian, magnetized, viscous, advective, coupled disc-jet model have been done in Reference [67]. A gravitational force was obtained using the pseudo-Newtonian potential. It is claimed that the maximum possible outflow power in this model is equal to $7.5 \times 10^{39} \mathrm{erg} / \mathrm{s}$ for a non-rotating stellar mass $\left(20 \mathrm{M}_{\odot}\right)$ ) black hole accreting at sub-Eddington accretion rate. The outflow power extracted from the disc is defined by the combination of mechanical, enthalpy, viscous and the Poynting parts. Hence, this scenario could provides an explanation of the nature of hard-state ULXs without introducing the existence of the missing class of intermediate mass BHs, nor with the super-Eddington accretion [67].

3. The main purpose of the paper [54] was to explain the bright/hard state observed during the bright/slow transition in the rising phases of transient outbursts of galactic BH candidates, like Cyg X-1. In the low/hard state, the X-ray spectrum is described by a hard power law with a high energy cut-off at $\sim 200 \mathrm{keV}$. With increasing luminosity, the source enters the bright/hard state, when the cut-off energy decreases down to $\sim 50 \mathrm{keV}$. This transition was explained in Reference [54] by formation of MAD, as a result of increasing of the accretion rate.

4. It is shown in Reference [68] that a variability timescale in the MAD model as defined in Reference [69] can reproduce the observed minimum variability timescale (MTS) - bulk Lorentz factor correlation as well as the MTS - luminosity (L) relation observed in the long GRB data and can also be applied/extended to AGN data.

\section{Conclusions}

During accretion of the matter with a large scale magnetic field into a $\mathrm{BH}$ the disk is formed around a black hole, which equilibrium is supported by the balance between a $\mathrm{BH}$ gravity and a magnetic pressure. The semi-analytical models of such disk a have been constructed in 70s [1,2]. An efficiency of transformation of the gravitational energy into the radiation in this model is very high, with $L / \dot{M} c^{2} \sim 0.5$. Such a high efficiency is connected with an action of the external field, which holds the disk from falling into $\mathrm{BH}$ up to smaller radius $\sim 1.5 r_{g}$. This model of accretion attracted attention in 2003 [25], where it was called as a magnetically arrested disk (MAD), reflecting a role of the magnetic field, holding the matter from falling into a $\mathrm{BH}$. Numerical simulations of the MAD model confirmed the semi-analytical results [1,2,25], being done in the framework of a pseudo-newtonian gravitational potential [4,47], as well as in the full GR consideration [3,5]. In an application of this model to accretion of the magnetized matter onto a NS it was called as magnetic-levitation accretion scenario [55]. Application of this model for an explanation of observational properties of X-pulsars of different types, and behavior of objects, containing a $\mathrm{BH}$, occurs to be rather promising.

Funding: This research received no external funding. 
Acknowledgments: This work was supported by Russian Science Foundation, Grant No. 18-12-00378.

Conflicts of Interest: The authors declare no conflict of interest. The founding sponsors had no role in the design of the study; in the collection, analyses or interpretation of data; in the writing of the manuscript and in the decision to publish the results.

\section{References}

1. Bisnovatyi-Kogan, G.S.; Ruzmaikin, A.A. The Accretion of Matter by a Collapsing Star in the Presence of a Magnetic Field. Astrophys. Space Sci. 1974, 28, 45-59. [CrossRef]

2. Bisnovatyi-Kogan, G.S.; Ruzmaikin, A.A. The accretion of matter by a collapsing star in the presence of a magnetic field. II - Selfconsistent stationary picture. Astrophys. Space Sci. 1976, 42, 401-424. [CrossRef]

3. Chael, A.; Narayan, R.; Johnson, M.D. Two-temperature, Magnetically Arrested Disc simulations of the jet from the supermassive black hole in M87. arXiv 2018, arXiv:1810.01983.

4. Igumenshchev, I.V. Magnetically arrested disks and the origin of poynting jets: A numerical study. Astrophys. J. 2008, 677, 317-326. [CrossRef]

5. Tchekhovskoy, A.; Narayan, R.; McKinney, J.C. Efficient generation of jets from magnetically arrested accretion on a rapidly spinning black hole. Mon. Not. R. Astron. Soc. Lett. 2011, 418, L79-L83. [CrossRef]

6. Chandrasekhar, S. The Mathematical Theory of Black Holes; Clarendon: Oxford, UK, 1983.

7. Novikov, I.D.; Frolov, V.P. Physics of Black Holes; Nauka: Moscow, Russia, 1986.

8. Zeldovich, Ya.B.; Novikov, I.D. Relativistic Astrophysics, Volume 1; Chicago University Press: Chicago, IL, USA, 1971.

9. Cherepashchuk, A.M. (Ed.) Catalog of Close Binary Systems at Late Evolutionary Stages; MGU Press: Moscow, Russia, 1988.

10. Cherepashchuk, A.M. Masses of black holes in binary systems. Soviet. Phys. Uspekhi 1996, 39, 759-780. [CrossRef]

11. Cherepashchuk, A.M. X-ray nova binary systems. Space Sci. Rev. 2000, 93, 473-580. [CrossRef]

12. Makishima, K.; Maejima, Y.; Mitsuda, K.; Bradt, H.V.; Remillard, R.A.; Tuohy, I.R.; Hoshi, R.; Nakagawa, M. Simultaneous X-ray and optical observations of GX 339-4 in an X-ray high state. Astrophys. J. 1986, 308, 635-643. [CrossRef]

13. Shvartsman, V.F. Halos around "Black Holes". Sov. Astron. 1971, 48, 479-488.

14. Kaplan, S.A.; Pikel'ner, S.B. The Interstellar Medium; Fizmatgiz: Moscow, Russia, 1963.

15. Bisnovatyi-Kogan, G.S. Magnetohydrodynamical processes near compact objects. Nuovo C. Riv. 1979, 2, 1-37. [CrossRef]

16. Anderson, J.; Cohen, J. Gravitational Collapse of Magnetic Neutron Stars. Astrophys. Space Sci. 1970, 9, 146-152. [CrossRef]

17. Ginzburg, V.L. The Magnetic Fields of Collapsing Masses and the Nature of Superstars. Sov. Phys. Dokl. 1964, 9, 329.

18. Balbus, S.A.; Hawley, J.F. Instability, turbulence, and enhanced transport in accretion disks. Rev. Mod. Phys. 1998, 70, 1-53. [CrossRef]

19. Bisnovatyi-Kogan, G.S.; Blinnikov, S.I. Disk accretion onto a black hole at subcritical luminosity. Astron. Astrophys. 1977, 59, 111-125.

20. Bisnovatyi-Kogan, G.S.; Lovelace, R.V.E.; Belinski, V.A. A Cosmic Battery Reconsidered. Astrophys. J. 2002, 580, 380-388. [CrossRef]

21. Contopoulos, I.; Kazanas, D. A Cosmic Battery. Astrophys. J. 1998, 508, 859-863. [CrossRef]

22. Amnuel, P.R.; Guseinov, O.H. The X-ray emission from neutron stars in the accretion process. Izv. Akad. Nauk Azerb. SSR 1968, 3, 70.

23. Pringle, J.; Rees, M. Accretion Disc Models for Compact X-ray Sources. Astron. Astrophys. 1972, 21, 1-9.

24. Shakura, N.I. Disk Model of Gas Accretion on a Relativistic Star in a Close Binary System. Sov. Astron. 1972, 49, 921-929. 
25. Narayan, R.; Igumenshchev, I.V.; Abramowicz, M.A. Magnetically Arrested Disk: An Energetically Efficient Accretion Flow. Publ. Astron. Soc. Jpn. 2003, 55, L69-L72. [CrossRef]

26. Lichnerowicz, A. Relativistic Hydrodynamics and Magnetohydrodynamics. 1967. Available online: https://www.osti. gov/biblio/5096753\#biblio-cite-chi (accessed on 10 June 2019).

27. Michel, F.C. Accretion of Matter by Condensed Objects. Astrophys. Space Sci. 1972, 15, 153-160. [CrossRef]

28. Landau, L.D.; Lifshitz, E.M. Field Theory; Nauka: Moscow, Russia, 1988.

29. Bondi, H. On spherically symmetrical accretion. Mon. Not. R. Astron. Soc. 1952, 112, 195-204 [CrossRef]

30. Shapiro, S.L.; Teukolsky, S.A. Black Holes, White Dwarfs, and Neutron Stars: The Physics of Compact Objects; Wiley-Interscience: New York, NY, USA, 1983.

31. Gammie, C.F. Efficiency of Magnetized Thin Accretion Disks in the Kerr Metric. Astrophys. J. 1999, 522, L57-L60. [CrossRef]

32. Mondal, T.; Mukhopadhyay, B. Magnetized advective accretion flows: Formation of magnetic barriers in magnetically arrested discs. Mon. Not. R. Astron. Soc. 2018, 476, 2396-2409. [CrossRef]

33. Bisnovatyi-Kogan, G.S.; Friedmann, A.M. A Mechanism for Emission of X Rays by a Neutron Star. Sov. Astron. 1969, 46, 721-724.

34. Bisnovatyi-Kogan, G.S. Stellar Physics: 2: Stellar Evolution and Stability; Astronomy and Astrophysics Library, Springer-Verlag: Berlin/Heidelberg, Germany, 2011.

35. Park, M.-G. Self-consistent models of spherical accretion onto black holes. I. One-temperature solutions. Astrophys. J. 1990, 354, 64-82 [CrossRef]

36. Park, M.-G. Self-consistent models of spherical accretion ontoblack holes. II. Two-temperature solutions with pairs. Astrophys. J. 1990, 354, 83-97. [CrossRef]

37. Bisnovatyi-Kogan, G.S.; Blinnikov, S.I. Spherical accretion onto compact X-ray source with preheating: No thermal limits for the luminosity. Mon. Not. R. Astron. Soc. 1980, 191, 711-719. [CrossRef]

38. Spiegel, E.E. The Gas Dynamics of Accretion. In Interstellar Gas Dynamics; Habing, H.J., Ed.; D. Reidel Publishing Company: Dordrecht-Holland, The Netherlands, 1970.

39. Bisnovatyi-Kogan, G.S.; Kazhdan, Y.M.; Klypin, A.A.; Lutskii, A.E.; Shakura, N.I. Accretion onto a rapidly moving gravitating center. Sov. Astron. 1979, 23, 201-205.

40. Bisnovatyi-Kogan, G.S.; Blinnikov, S.I. The Equilibrium, Stability and Evolution of a Rotating Magnetized Gaseous Disk. Astrophys. Space Sci. 1972, 19, 119-144. [CrossRef]

41. Pikelner, S.B. Foundations of Space Electrodynamics; Nauka: Moscow, Russia, 1966.

42. Bisnovatyi-Kogan, G.S. Stellar Physics. Vol.1: Fundamental Concepts and Stellar Equilibrium; Astronomy and Astrophysics Library; Springer: Berlin, Germany, 2001.

43. Bisnovatyi-Kogan, G.S.; Blinnikov, S.I. A hot corona around a black-hole accretion disk as a model for Cygnus X-1. Sov. Astron. Lett. 1976, 2, 191-193.

44. Blandford, R.D.; Znajek, R.L. Electromagnetic extraction of energy from Kerr black holes. Mon. Not. R. Astron. Soc. 1977, 179, 433-456. [CrossRef]

45. Lovelace, R.V.E. Dynamo model of double radio sources. Nature 1976, 262, 649-652. [CrossRef]

46. Goldreich, P.; Julian, W.H. Pulsar Electrodynamics. Astrophys. J. 1969, 157, 869-880. [CrossRef]

47. Paczyński, B.; Wiita, P.J. Thick accretion disks and supercritical luminosities. Astron. Astrophys. 1980, 88, $23-31$.

48. Igumenshchev, I.V.; Narayan, R.; Abramowicz, M.A. Three-dimensional Magnetohydrodynamic Simulations of Radiatively Inefficient Accretion Flows. Astrophys. J. 2003, 592, 1042-1059. [CrossRef]

49. Bisnovatyi-Kogan, G.S.; Lovelace, R.V.E. Influence of ohmic heating on advection-dominated accretion flows. Astrophys. J. 1997, 486, L43-L48. [CrossRef]

50. Colella, P.; Woodward, P.R. The Piecewise Parabolic Method (PPM) for Gas-Dynamical Simulations. J. Comput. Phys. 1984, 54, 174-201. [CrossRef]

51. Machida, M.; Nakamura, K.E.; Matsumoto, R. Formation of Magnetically Supported Disks during Hard-to-Soft Transitions in Black Hole Accretion Flows. Publ. Astron. Soc. Jpn. 2006, 58, 193-202. [CrossRef]

52. Penrose, R. Gravitational Collapse: The Role of General Relativity. Nuovo C. Riv. Ser. 1969, 1, 252. 
53. Ruffini, R.; Wilson, J.R. Relativistic magnetohydrodynamical effects of plasma accreting into a black hole. Phys. Rev. D 1975, 12, 2959-2962. [CrossRef]

54. Oda, H.; Machida, M.; Nakamura, K.E.; Matsumoto, R. Thermal Equilibria of Optically Thin, Magnetically Supported, Two-temperature, Black Hole Accretion Disks. Astrophys. J. 2010, 712, 639-652. [CrossRef]

55. Ikhsanov, N.R.; Kim, V.Y.; Beskrovnaya, N.G. A Scenario of the Formation of Isolated X-ray Pulsars with Anomalously Long Period. Astron. Rep. 2015, 59, 25-32. [CrossRef]

56. Ikhsanov, N.R.; Beskrovnaya, N.G. Signs of Magnetic Accretion in X-ray Pulsars. Astron. Rep. 2012, 56, 589-594. [CrossRef]

57. Ikhsanov, N.R.; Finger, M.H. Signs of magnetic accretion in the X-ray pulsar binary GX 301-2. Astrophys. J. 2012, 753, 1. [CrossRef]

58. Ikhsanov, N.; Beskrovnaya, N.; Likh, Y. Evidence for magneto-levitation accretion in long-period X-ray pulsars. Int. J. Mod. Phys. Conf. Ser. 2014, 28, 1460187. [CrossRef]

59. Ikhsanov, N.R.; Likh, Y.S.; Beskrovnaya, N.G. Spin Evolution of Long-Period X-ray Pulsars Astron. Rep. 2014, 58, 376-385.

60. Ikhsanov, N.R.; Beskrovnaya, N.G. The Spin-Down Mechanism of the X-ray Pulsar 4U 2206+54. Astron. Rep. 2013, 57, 287-293. [CrossRef]

61. Ikhsanov, N.R. Signs of magnetic accretion in the young Be/X-ray pulsar SXP 1062. Mon. Not. R. Astron. Soc. 2012, 424, L39-L43. [CrossRef]

62. Ikhsanov, N.R.; Kim, V.Y.; Beskrovnaya, N.G.; Pustil'nik, L.A. A new look at the origin of the $6.67 \mathrm{hr}$ period X-ray pulsar 1E 161348-5055 Astrophys. Space Sci. 2013, 346, 105-109.

63. Bisnovatyi-Kogan, G.S.; Ikhsanov, N.R. A New Look at Anomalous X-ray Pulsars. Astron. Rep. 2014, 58, $217-227$. [CrossRef]

64. Bisnovatyi-Kogan, G.S.; Chechetkin, V.M. Nucleosynthesis in Supernova Outbursts and the Chemical Composition of the Envelopes of Neutron Stars. Astrophys. Space Sci. 1974, 26, 25-46. [CrossRef]

65. Bisnovatyi-Kogan, G.S.; Chechetkin, V.M. Nonequilibrium shells of neutron stars and their role in sustaining X-ray emission and nucleosynthesis. Sov. Phys. Uspekhi 1979, 22, 89-108. [CrossRef]

66. Mondal, T.; Mukhopadhyay, B. FSRQ/BL Lac dichotomy as the magnetized advective accretion process around black holes: A unified classification of blazars. Mon. Not. R. Astron. Soc. 2019, 486, 3465-3472. [CrossRef]

67. Mondal, T.; Mukhopadhyay, B. Ultraluminous X-ray sources as magnetically powered sub-Eddington advective accretion flows around stellar mass black holes. Mon. Not. R. Astron. Soc. 2019, 482, L24-L28. [CrossRef]

68. Lloyd-Ronning, N.; Lei, W.; Xie, W. An MAD explanation for the correlation between bulk Lorentz factor and minimum variability time-scale. Mon. Not. R. Astron. Soc. 2018, 478, 3525-3529. [CrossRef]

69. Lloyd-Ronning, N.M.; Dolence, J.C.; Fryer, C.L. A MAD model for gamma-ray burst variability. Month. Not. R. Astron. Soc. 2016, 461, 1045-1052. [CrossRef]

(C) 2019 by the authors. Licensee MDPI, Basel, Switzerland. This article is an open access article distributed under the terms and conditions of the Creative Commons Attribution (CC BY) license (http:/ / creativecommons.org/licenses/by/4.0/). 